\title{
Evolution of transverse flow and effective temperatures in the parton phase from a multi-phase transport model
}

\author{
Zi-Wei Lin* \\ Department of Physics, East Carolina University, \\ C-209 Howell Science Complex, Greenville, NC 27858
}

\begin{abstract}
I study the space-time evolution of transverse flow and effective temperatures in the dense parton phase with the string melting version of a multi-phase transport model. Parameters of the model are first constrained to reproduce the bulk data on the rapidity density, $p_{\mathrm{T}}$ spectrum and elliptic flow at low $p_{\mathrm{T}}$ for central and mid-central $\mathrm{Au}+\mathrm{Au}$ collisions at $200 \mathrm{~A}$ $\mathrm{GeV}$ and $\mathrm{Pb}+\mathrm{Pb}$ collisions at $2760 A \mathrm{GeV}$. I then calculate the transverse flow and effective temperatures in volume cells within mid-spacetime-rapidity $|\eta|<1 / 2$. I find that the effective temperatures extracted from different variables, which are all evaluated in the rest frame of a volume cell, can be very different; this indicates that the parton system in the model is not in full chemical or thermal equilibrium locally, even after averaging over many events. In particular, the effective temperatures extracted from the parton energy density or number density are often quite different than those extracted from the parton mean $p_{\mathrm{T}}$ or mean energy. For these collisions in general, effective temperatures extracted from the parton energy density or number density are higher than those extracted from the parton mean $p_{\mathrm{T}}$ in the inner part of the overlap volume, while the opposite occurs in the outer part of the overlap volume. I argue that this indicates that the dense parton matter in the inner part of the overlap volume is over-populated; I also find that all cells with energy density above 1 $\mathrm{GeV} / \mathrm{fm}^{3}$ are over-populated after a couple of $\mathrm{fm} / c$.
\end{abstract}

PACS numbers: 12.38.Mh, 25.75.Ld, 25.75.Nq,24.10.Lx

\footnotetext{
*linz@ecu.edu
} 


\section{INTRODUCTION}

A dense matter consisting of partonic degrees of freedom, often called the quarkgluon plasma (QGP), has been created in ultra-relativistic heavy ion collisions at the Relativistic Heavy Ion Collider (RHIC) and the Large Hadron Collider (LHC). Its properties such as the color glass condensate initial condition, the degree of equilibration, event-by-event fluctuations, and coupling with high-momentum partons are being extensively studied [1-4]. Simulations of these collisions with hydrodynamic codes [5] 8 , transport models [9-11], or hybrid models [12 14] are able to produce the full evolution history of dense matter and are very useful for the studies of the quark-gluon plasma properties. In this paper I investigate the evolution of the parton matter at mid-spacetime-rapidity, including its transverse flow and effective temperatures. The string melting version of the AMPT model [10] is used here, where excited hadronic strings in the overlap volume are converted into partons via the intermediate step of decomposing hadrons that would have been produced by the Lund string fragmentation process [10, 15].

The default version of the AMPT model [16, 17] was first constructed to simulate relativistic heavy ion collisions, and its key parameters were determined to fit the yields and $p_{\mathrm{T}}$ spectra of particles in pp collisions at various energies and heavy ion collisions up to SPS energies. Hadronization in the default AMPT model is described by the Lund string model [18], where one assumes that a string fragments into quarkantiquark pairs with a Gaussian distribution in transverse momentum. Hadrons are formed from these quarks and antiquarks, with its longitudinal momentum given by the Lund symmetric fragmentation function $f(z) \propto z^{-1}(1-z)^{a} \exp \left(-b m_{\mathrm{T}}^{2} / z\right)$, where $z$ represents the light-cone momentum fraction of the produced hadron with respect to that of the fragmenting string and $m_{\mathrm{T}}$ is the transverse mass of the hadron. It was found [16, 17] that the default HIJING values for the Lund string fragmentation parameters $\left(a=0.5\right.$ and $b=0.9 \mathrm{GeV}^{-2}$ ), which work well for pp collisions, led to

too small a charged particle yield in central $\mathrm{Pb}+\mathrm{Pb}$ collisions at the SPS energy of $E_{\mathrm{LAB}}=158 A \mathrm{GeV}$. Therefore modified values of $a=2.2$ and $b=0.5 \mathrm{GeV}^{-2}$ were used in order to fit the charged particle yield in $\mathrm{Pb}+\mathrm{Pb}$ collisions at SPS. For heavy ion 
collisions at higher energies such as RHIC energies, the default version of the AMPT model with these parameters values was found to reasonably fit $\mathrm{dN} / \mathrm{d} \eta, \mathrm{dN} / \mathrm{dy}$ and the $p_{\mathrm{T}}$ spectra in heavy ion collisions, although it under-estimates the elliptic flow. On the other hand, the string melting version of the AMPT model (AMPT-SM) [10, 15], due to its dense parton phase, reasonably fits the elliptic flow and two-pion HBT in heavy ion collisions; but it does not reproduce well $\mathrm{dN} / \mathrm{d} \eta, \mathrm{dN} / \mathrm{dy}$ and the $p_{\mathrm{T}}$ spectra (when using the same parameters as in the default version). In particular, the AMPTSM model significantly over-estimates the charged particle yield while under-estimates the slopes of the $p_{\mathrm{T}}$ spectra [10]. In an earlier attempt to reproduce data in $\mathrm{Pb}+\mathrm{Pb}$ collisions at LHC energies with the AMPT-SM model, the default HIJING values for the Lund string fragmentation parameters were used [19] together with the strong coupling constant $\alpha_{s}=0.33$ (instead of 0.47 ); there the model reasonably reproduced the yield and elliptic flow of charged particles but underestimated the $p_{\mathrm{T}}$ spectrum (except at low $p_{\mathrm{T}}$ ).

In this study I use the AMPT-SM model to study the evolution of the dense parton phase. I first tune the key parameters of the model to reproduce the pion and kaon yields, $p_{\mathrm{T}}$ spectra, and elliptic flows at low $p_{\mathrm{T}}$ (below $\sim 2 \mathrm{GeV} / c$ ) in central and mid-central $\mathrm{Au}+\mathrm{Au}$ collisions at the RHIC energy of $200 \mathrm{~A} \mathrm{GeV}$ and $\mathrm{Pb}+\mathrm{Pb}$ collisions at the LHC energy of $2760 A \mathrm{GeV}$. I then study the evolution of transverse flow and effective temperature in the parton phase. In the analysis, the reaction volume within $|\eta|<1 / 2$ is divided into cells with a transverse width of $1 \mathrm{fm}$. In order to have enough statistics for the analysis of each volume cell, I choose to study event-averaged quantities and thus neglect event-by-event fluctuations in this study. For each collision energy and centrality, I run hundreds to thousands of events at the same impact parameter range and then calculate event-averaged quantities at functions of time and transverse location. Note that the AMPT-SM model assigns a formation time to each parton produced from string melting [10], and only partons that have formed by a global time $t$ are included in the analysis for that time. The full space-time evolutions of the parton matter from this study have been posted online ${ }^{1}$ and may be used as the bulk matter

\footnotetext{
${ }^{1}$ Grid data files of the space-time evolution of the parton matter from this study have been posted
} 
background within which other probes such as jet propagation and interactions can be studied [22, 23].

\section{FITTING THE BULK DATA WITH THE STRING MELTING VERSION OF AMPT}

Since the purpose of the study is to obtain the space-time evolution of the dense parton matter, I first constrain the model parameters by fitting low $p_{\mathrm{T}}$ data in heavy ion collisions at RHIC and LHC energies. To fit the low $p_{\mathrm{T}}$ data in central and midcentral $\mathrm{Au}+\mathrm{Au}$ collisions at the top RHIC energy, I find that I need to set the values of the Lund string fragmentation parameters to $a=0.55, b=0.15 \mathrm{GeV}^{-2}$. Note that I use a lower $b$ value than in previous studies [10, 15-17, 19] in order to simultaneously fit the rapidity density, $p_{\mathrm{T}}$ spectrum and elliptic flow of pions and kaons at low $p_{\mathrm{T}}$ with the string melting version of the AMPT model. Also note that the effective string tension, as given by $\kappa \propto 1 /[b(2+a)]$ [10], will change when the Lund string fragmentation parameters are modified; and a smaller $b$ value will lead to a larger mean transverse momentum of the initial hadrons. In addition, the AMPT model assumes that the relative production of strange to non-strange quarks increases with the effective string tension [10]. Since the above string tension relation diverges as $b \rightarrow 0$ and this study uses a small $b$ value to enable the AMPT-SM results to fit the data, I put an upper limit of 0.40 on the relative production of strange to non-strange quarks in AMPT. For $\mathrm{Pb}+\mathrm{Pb}$ collisions at the LHC energy, I use $a=0.30$ (and $b=0.15 \mathrm{GeV}^{-2}$ ) in order to fit the ALICE data [24]. I take $\alpha_{s}=0.33$ and a parton cross section of $3 \mathrm{mb}$ for all simulations in this study. There may be other AMPT parameter sets that could reasonably fit the data. For example, I find that the Lund $a$ value of 0.55 used for fitting the RHIC data gives a reasonable but less satisfactory fit of the ALICE data than the $a$ value of 0.30 . One may also use a lower value of the strong coupling $\alpha_{s}$ at LHC than at RHIC [3] and tune other AMPT parameters to obtain such a simultaneous fit to data.

20]; the link has also been posted at the JET Collaboration wiki page 21]. 


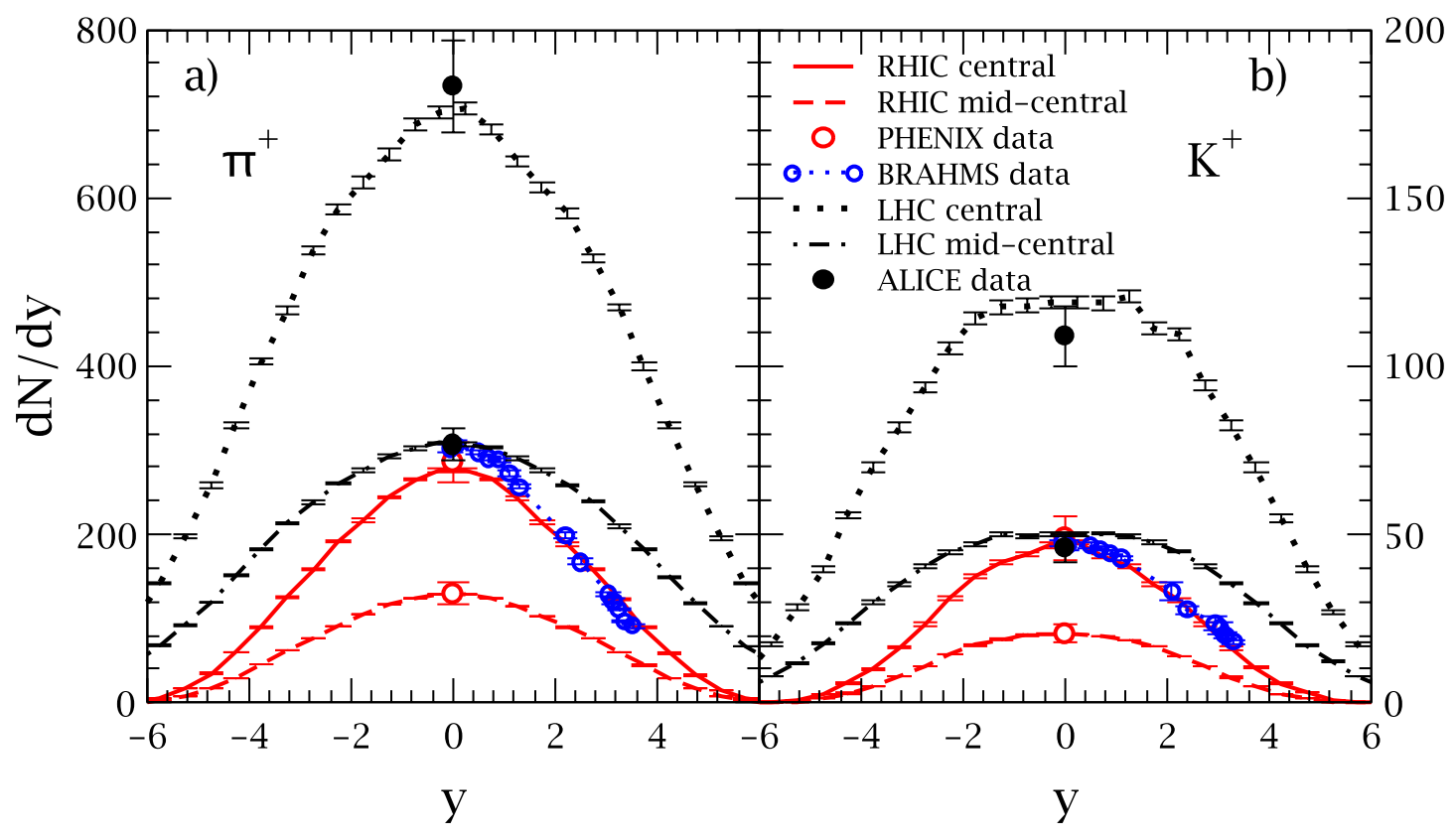

FIG. 1: (Color online) AMPT-SM results for central and mid-central Au+Au collisions at $200 A \mathrm{GeV}$ and $\mathrm{Pb}+\mathrm{Pb}$ collisions at $2760 A \mathrm{GeV}$ in comparison with experimental data for 0-5\% and 20-30\% centralities: a) dN/dy of $\pi^{+}$, and b) dN/dy of $K^{+}$.

The AMPT-SM results shown in this study include four collision systems: RHIC central refers to $\mathrm{Au}+\mathrm{Au}$ events at $200 A \mathrm{GeV}$ with $b<3$ fm that represent the $0-5 \%$ centrality, RHIC mid-central refers to $\mathrm{Au}+\mathrm{Au}$ events at $200 A \mathrm{GeV}$ with $b=7.3 \mathrm{fm}$ that represent the $20-30 \%$ centrality [25], LHC central refers to $\mathrm{Pb}+\mathrm{Pb}$ events at $2760 \mathrm{~A}$ $\mathrm{GeV}$ with $b<3.5 \mathrm{fm}$ that represent the $0-5 \%$ centrality, and LHC mid-central refers to $\mathrm{Pb}+\mathrm{Pb}$ events at $2760 A \mathrm{GeV}$ with $b=7.8 \mathrm{fm}$ that represent the $20-30 \%$ centrality [26]. Since each volume cell within $|\eta|<1 / 2$ has a transverse width of $1.0 \mathrm{fm}$ along both the $x$ - and $y$-axis, the cell at $x=3 \mathrm{fm} \& y=0 \mathrm{fm}$ then refers to the volume within $2.5<x<3.5 \mathrm{fm} \&-0.5<y<0.5 \mathrm{fm} \&|\eta|<1 / 2$, for example.

The comparisons between AMPT-SM results and the experimental data on particle dN/dy are shown in Fig. 1 1 for $\pi^{+}$and in Fig. $1 \mathrm{~b}$ for $K^{+}$, where we see good agreements between the model results and the PHENIX [27] and ALICE data 24] at mid-rapidity in both central and mid-central events at RHIC and LHC energies. Reasonable agreements are also seen in comparison with the BRAHMS [28] data at different rapidities 


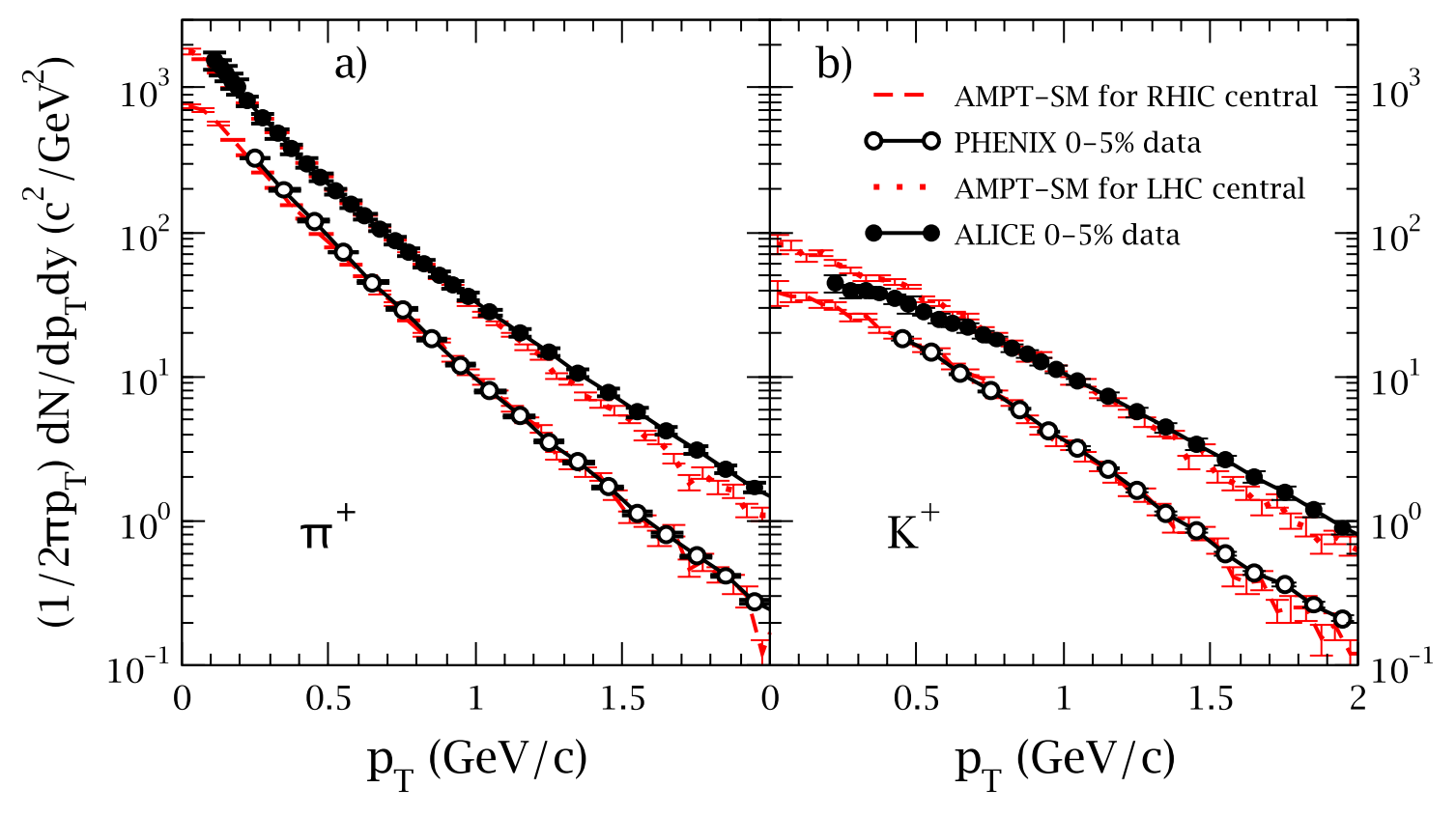

FIG. 2: (Color online) $p_{\mathrm{T}}$ spectra of $\pi^{+}$and $K^{+}$at mid-rapidity in central collisions from AMPT-SM in comparison with experimental data for 0-5\% centrality.

in $0-5 \%$ central $\mathrm{Au}+\mathrm{Au}$ collisions at $200 \mathrm{~A} \mathrm{GeV}$. The comparisons of the $\pi^{+}$and $K^{+}$ $p_{\mathrm{T}}$ spectra at mid-rapidity are shown in Fig. 2a and Fig. 2b respectively for central $\mathrm{Au}+\mathrm{Au}$ collisions at the RHIC energy and central $\mathrm{Pb}+\mathrm{Pb}$ collisions at the LHC energy, where the model roughly reproduces the observed $p_{\mathrm{T}}$ spectra below $2 \mathrm{GeV} / c$.

Fig. 3 shows the comparisons of $v_{2}\{\mathrm{EP}\}$ of charged pions and kaons. We see that the AMPT-SM model is also able to roughly reproduce the pion and kaon elliptic flow data on $v_{2}\{\mathrm{EP}\}$ [29] at low- $p_{\mathrm{T}}$. Note that the correction factor for the event plane resolution $\operatorname{Res}\left\{2 \Phi_{2}\right\}$ [30] in the AMPT-SM results is calculated using particles within rapidities $-2.8<y<-1$ and within $1<y<2.8$.

\section{SPACE-TIME EVOLUTION OF TRANSVERSE FLOW}

For each volume cell within $|\eta|<1 / 2$, I calculate the flow as $\vec{\beta}=\left(\sum_{i} \vec{p}_{i}\right) /\left(\sum_{i} E_{i}\right)$, where the sum over index $i$ takes into account all formed partons in the cell from all events of a given collision system. Fig. 4 shows the time evolutions of the transverse flow 




FIG. 3: (Color online) Elliptic flow $v_{2}\{\mathrm{EP}\}$ at mid-rapidity in mid-central collisions from AMPT-SM (curves without circles) in comparison with experimental data for $20-30 \%$ centrality (circles): a) for charged pions, and b) for charged kaons.

of partons in two cells in central $\mathrm{Au}+\mathrm{Au}$ collisions at $200 \mathrm{~A} \mathrm{GeV}$ : one cell at $x=3 \mathrm{fm}$ $\& y=0 \mathrm{fm}$ and the other cell at $x=7 \mathrm{fm} \& y=0 \mathrm{fm}$. The flows along the $y$-direction in both cells here are essentially zero due to the symmetry after averaging over many events. For the flow along the $x$-direction, however, we see that both the profile and magnitude depend significantly on the location even for these central collisions: the flow in the cell closer to the center of the overlap volume develops gradually and then decreases at later times, while the flow in the cell farther away from the center develops very fast and reaches a bigger magnitude. Note that in the AMPT model the centers of the two incoming nuclei are essentially at $x=b / 2 \& y=0 \mathrm{fm}$ and $x=-b / 2 \& y=0$ $\mathrm{fm}$, respectively. As a result, the center of the overlap region of each $\mathrm{Au}+\mathrm{Au}$ or $\mathrm{Pb}+\mathrm{Pb}$ event is at $x=0 \mathrm{fm} \& y=0 \mathrm{fm}$, when event-by-event fluctuations are neglected or when the calculation averages over many events at the same impact parameter.

Fig. 5 shows transverse flows along the $x$-direction in cells within $-0.5<y<0.5 \mathrm{fm}$ 


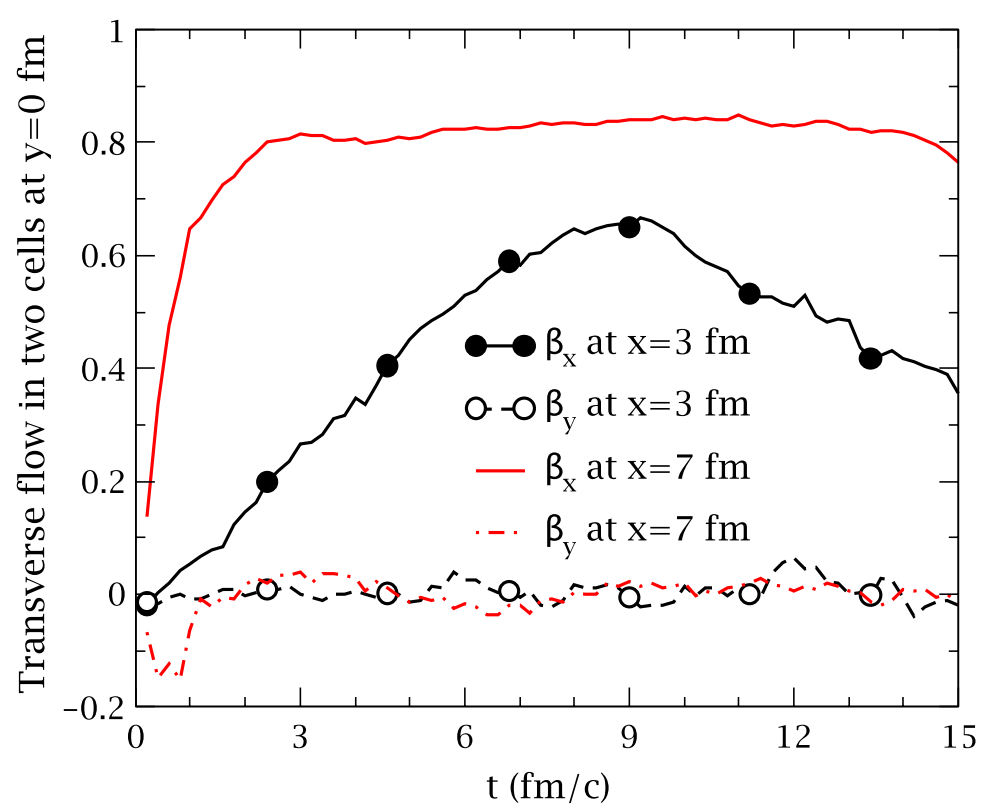

FIG. 4: (Color online) Transverse flow of partons in the cell at $x=3 \mathrm{fm} \& y=0 \mathrm{fm}$ and in the cell at $x=7 \mathrm{fm} \& y=0 \mathrm{fm}$ as functions of time in central $\mathrm{Au}+\mathrm{Au}$ collisions at $200 \mathrm{~A}$ GeV.

at different locations along the $x$-axis at various times in central $\mathrm{Au}+\mathrm{Au}$ collisions at RHIC. We see that the flow is initially very small at the early time $t=0.2 \mathrm{fm} / c$ and then develops rather fast, while for the inner part of the overlap region $(-4<x<4 \mathrm{fm})$ the flow decreases appreciably later in the evolution (after $t \sim 8 \mathrm{fm} / c$ ). We also see that in general the flow magnitude is bigger the further away from the overlap center in these central collisions regardless of time $t$ shown in the figure.

\section{SPACE-TIME EVOLUTION OF TEMPERATURE}

Partons in a given volume of a heavy ion collision, even after being averaged over many events at the same impact parameter, may not be in full thermal equilibrium or full chemical equilibrium. If this is the case, the "temperature" for such a local parton system will only be an effective temperature, which value depends on the variable that it is extracted from. In this study I use different variables in the rest frame of a cell, such as the parton mean $p_{\mathrm{T}}$, number density and energy density, to extract the effective 


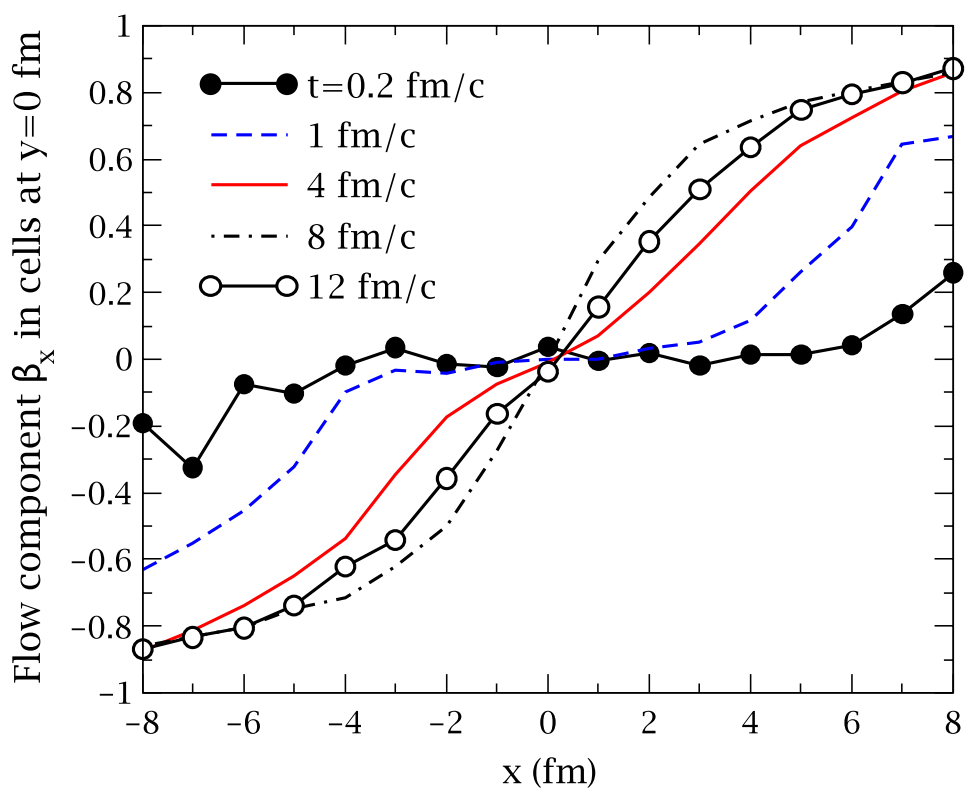

FIG. 5: (Color online) Flow along the $x$-direction as functions of $x$ at different times in cells within $-0.5<y<0.5 \mathrm{fm}$ in central $\mathrm{Au}+\mathrm{Au}$ collisions at $200 \mathrm{~A} \mathrm{GeV}$.

temperature of partons in the cell.

To extract the effective temperature from the mean momentum of partons in the rest frame of a cell, I use the relations for a massless parton gas in thermal equilibrium as given by the Boltzmann distribution to get

$$
T_{\langle p\rangle}=\frac{\langle p\rangle}{3}, T_{\left\langle p_{\mathrm{T}}\right\rangle}=\frac{4\left\langle p_{\mathrm{T}}\right\rangle}{3 \pi}, T_{\left\langle p_{\mathrm{T}}^{2}\right\rangle}=\sqrt{\frac{\left\langle p_{\mathrm{T}}^{2}\right\rangle}{8}} .
$$

In the above, the bracket represents the mean value of a variable, $p$ represents the magnitude of the parton 3 -momentum, and $p_{\mathrm{T}}$ represents the parton transverse momentum. Note that quark masses in the parton phase of the AMPT model are current quark masses [10]. Also, the parton phase in the AMPT model does not include the effect of quantum statistics, and the Boltzmann distribution is assumed in the formulae and corresponding curves in this study unless specified otherwise.

I also extract the effective temperature from the number density or energy density of partons in the rest frame of a cell. For this purpose I use the following relations between the densities and temperature $T$ for a massless quark-gluon plasma in full 
chemical and thermal equilibrium as given by the Boltzmann distribution:

$$
n=g_{\mathrm{B}} \frac{T^{3}}{\pi^{2}}, \epsilon_{\mathrm{T}}=3 g_{\mathrm{B}} \frac{T^{4}}{4 \pi}, \epsilon=3 g_{\mathrm{B}} \frac{T^{4}}{\pi^{2}} .
$$

In the above, $n$ is the parton number density, $\epsilon_{\mathrm{T}}$ is the transverse energy density, $\epsilon$ is the energy density, $g_{\mathrm{B}} \equiv 4\left(4+3 N_{f}\right)$ is the total degeneracy factor of QGP when using Boltzmann distributions, and $N_{f}$ represents the number of relevant quark flavors. I then have the following equations for the corresponding effective temperatures:

$$
\begin{gathered}
T_{n}=168 . \mathrm{MeV}\left(\frac{n \mathrm{fm}^{3}}{1+3 N_{f} / 4}\right)^{1 / 3}, \\
T_{\epsilon_{\mathrm{T}}}=212 . \mathrm{MeV}\left(\frac{\epsilon_{\mathrm{T}} \mathrm{fm}^{3} / \mathrm{GeV}}{1+3 N_{f} / 4}\right)^{1 / 4}, \\
T_{\epsilon}=199 . \mathrm{MeV}\left(\frac{\epsilon \mathrm{fm}^{3} / \mathrm{GeV}}{1+3 N_{f} / 4}\right)^{1 / 4} .
\end{gathered}
$$

I use the above relations to extract effective temperatures, and I take $N_{f}=3$ throughout this study. Note that using Bose-Einstein and Fermi-Dirac distributions for a massless quark-gluon plasma would lead to $n=\left(16+9 N_{f}\right) \zeta(3) T^{3} / \pi^{2}$ and $\epsilon=\pi^{2}\left(16+10.5 N_{f}\right) T^{4} / 30$, where $\zeta(3) \simeq 1.20$ is a Riemann zeta function. For $N_{f}=3$ they would give $n \simeq 5.24 T^{3}$ and $\epsilon \simeq 15.6 T^{4}$, which are very close to the relations $n \simeq 5.27 T^{3}$ and $\epsilon \simeq 15.8 T^{4}$ when the Boltzmann distribution is used for massless partons as done in Eqs. 2, 3 .

\section{A. Time evolution of parton densities and mean momentum}

I first examine the parton variables evaluated in the rest frame of the cells, which I shall use to extract the effective temperatures. The left panel of Fig. 6 shows the time evolutions of different variables in the center cell (at $x=0 \mathrm{fm} \& y=0 \mathrm{fm}$ ) in central $\mathrm{Au}+\mathrm{Au}$ collisions at $200 \mathrm{~A} \mathrm{GeV}$; they include the parton energy density (filled circles), number density (solid curve), mean $p_{\mathrm{T}}$ (open circles), mean transverse energy (dashed curve), and mean energy $\langle E\rangle$ (dot-dashed curve). The parton number density is seen to decrease much faster with time than the parton mean $p_{\mathrm{T}}$ or mean energy here. Consequently the parton energy density decreases even faster with time than the parton 

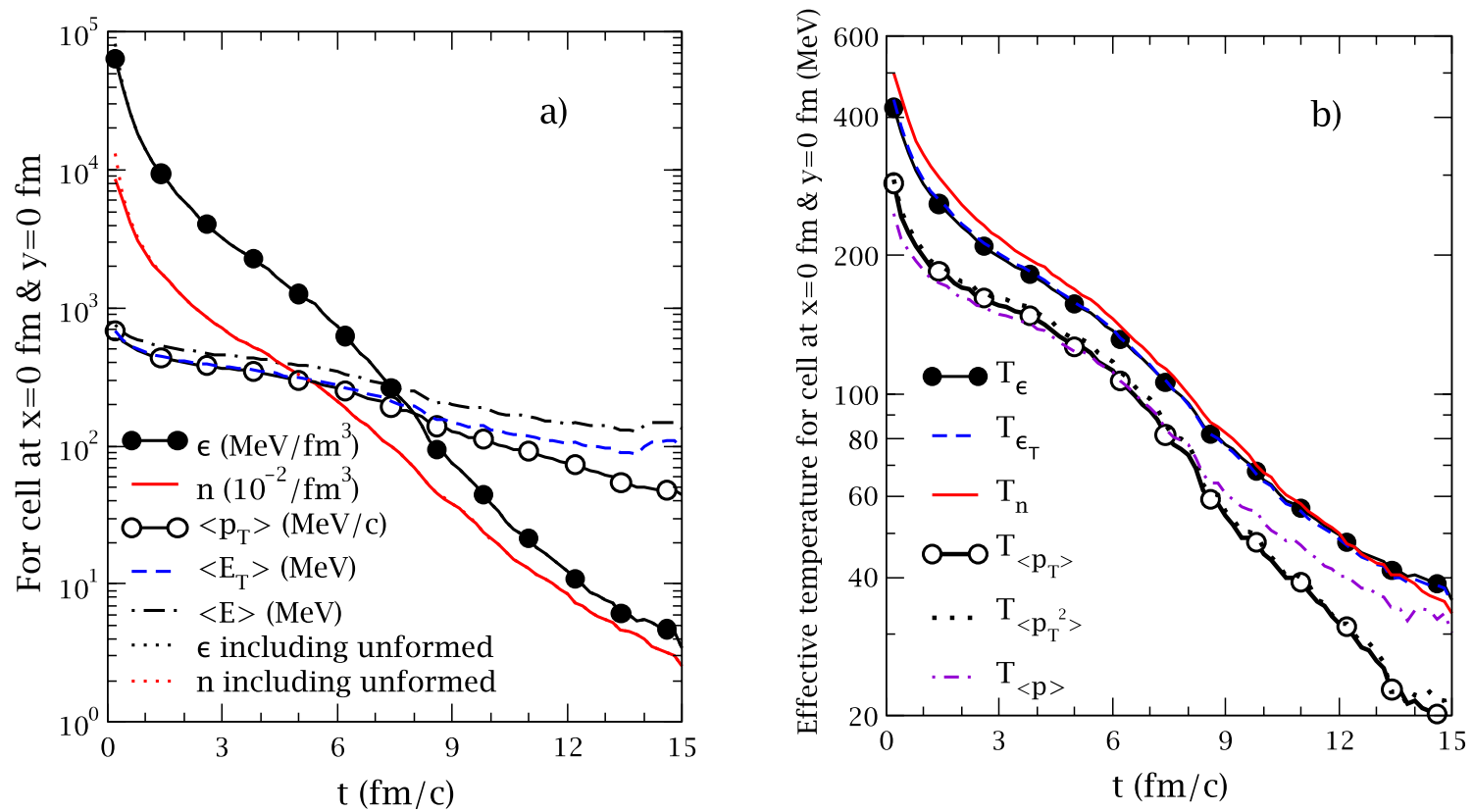

FIG. 6: (Color online) AMPT-SM results for partons in the rest frame of the center cell in central $\mathrm{Au}+\mathrm{Au}$ collisions at $200 \mathrm{~A} \mathrm{GeV}$ : a) time evolutions of the energy density, number density, mean $p_{\mathrm{T}}$, mean transverse energy, and mean energy; and b) time evolutions of effective temperatures extracted from different variables.

mean energy, which is expected since $\epsilon \equiv n\langle E\rangle$. We also see that the mean $p_{\mathrm{T}}$ and mean transverse energy are essentially the same at early times, and then they separate at late times when parton mass (especially the strange quark mass) plays a bigger role as the mean $p_{\mathrm{T}}$ becomes smaller. Furthermore, the amount by which the parton mean energy is above the mean transverse energy reflects the contribution from the squared longitudinal momentum $p_{\mathrm{z}}^{2}$ of partons under consideration (i.e. within $|\eta|<1 / 2$ ). In addition, the two dotted lines in the left panel of Fig. 6 show the parton energy density and number density when all partons (including unformed partons) are included. We see that unformed partons contribute little to the energy density and number density except for the earliest stage $(t<0.4 \mathrm{fm} / c$ for partons at mid-spacetime-rapidity in this study), and their relative contribution to the energy density is less than that to the number density since the average formation time of hard partons is shorter than that of soft partons in the string melting version of the AMPT model [10]. 


\section{B. Time evolution of effective temperatures}

The right panel of Fig. 6 shows time evolutions of effective temperatures extracted from different variables via Eqs. (1-3) for the center cell in central $\mathrm{Au}+\mathrm{Au}$ collisions at $200 \mathrm{~A} \mathrm{GeV}$. Here we see that the effective temperatures extracted from the parton energy density (filled circles), number density (solid curve), and transverse energy density (dashed curve) are all significantly higher than those extracted from the shown mean momentum variables, i.e., those extracted from the parton mean $p_{\mathrm{T}}$ (open circles), mean $p_{\mathrm{T}}^{2}$ (dotted curve), and mean 3-momentum (dot-dashed curve). If the local parton system in this cell were in full chemical and thermal equilibrium, temperature values extracted from these different variables at a given time would all be the same and we would only see one curve. Thus the different effective temperatures reflect the nonequilibrium nature of the local parton system. We also see that effective temperature $T_{\epsilon}$ extracted from the parton energy density is very close to $T_{\epsilon_{\mathrm{T}}}$ that is extracted from the parton transverse energy density throughout the time evolution. While the

effective temperature $T_{\left\langle p_{\mathrm{T}}\right\rangle}$ extracted from the parton mean $p_{\mathrm{T}}$ is very close to $T_{\left\langle p_{\mathrm{T}}^{2}\right\rangle}$ that is extracted from the parton mean $p_{\mathrm{T}}^{2}$, it is higher than $T_{\langle p\rangle}$ (extracted from the parton mean 3-momentum) at early times and then lower than $T_{\langle p\rangle}$ at late times. This suggests that the mean squared longitudinal momentum $p_{\mathrm{z}}^{2}$ is lower than the mean squared momentum along a transverse direction at early times but higher than that at later times; this is consistent with an earlier study on the pressure anisotropy in the AMPT model [31], where the ratio of the longitudinal pressure over the transverse pressure starts initially below one but then quickly exceeds one after a time of several $\mathrm{fm} / c$. Recently pressure anisotropy has also been considered within the hydrodynamic framework 32 34].

From the right panel of Fig. 6, we also observe that $T_{\epsilon}$ is mostly between $T_{\left\langle p_{\mathrm{T}}\right\rangle}$ and $T_{n}$, the effective temperature extracted from the parton number density, while being close to $T_{n}$. This can be understood in the following. Relation $\epsilon_{\mathrm{T}}=n\left\langle E_{\mathrm{T}}\right\rangle$ gives

$$
T_{\epsilon_{\mathrm{T}}}^{4}=T_{n}^{3} T_{\left\langle E_{\mathrm{T}}\right\rangle}
$$

Since $T_{\epsilon_{\mathrm{T}}} \simeq T_{\epsilon}$ as shown in the right panel of Fig. 6 and at high densities $\left\langle E_{\mathrm{T}}\right\rangle \simeq\left\langle p_{\mathrm{T}}\right\rangle$ 
as shown in the left panel of Fig. 6, I can write

$$
T_{\epsilon}^{4} \simeq T_{n}^{3} T_{\left\langle p_{\mathrm{T}}\right\rangle}
$$

I find that the above relation for $T_{\epsilon}$ is a good approximation at high densities: for all cells within $|\eta|<1 / 2$ in all four collisions systems that I considered, the actual $T_{\epsilon}$ value is found to be always within $6 \%$ of the value as given by Eq. (5) if $T_{\epsilon}>100 \mathrm{MeV}$ in the cell.

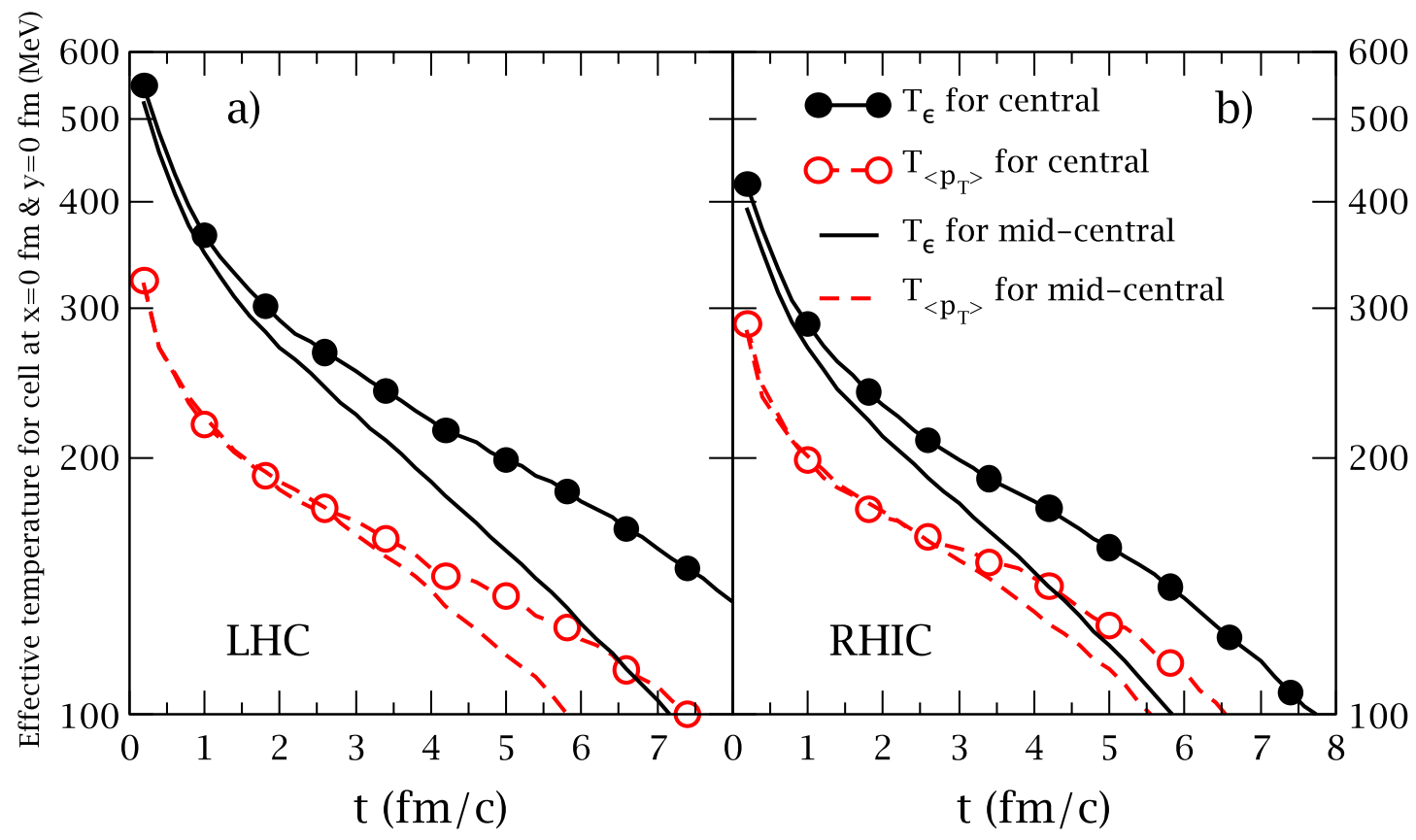

FIG. 7: (Color online) AMPT-SM results on effective temperatures as functions of time for the center cell in a) $\mathrm{Pb}+\mathrm{Pb}$ collisions at $2760 A \mathrm{GeV}$, and b) $\mathrm{Au}+\mathrm{Au}$ collisions at $200 \mathrm{~A} \mathrm{GeV}$.

Fig. 7] shows the time evolutions of $T_{\epsilon}$ and $T_{\left\langle p_{\mathrm{T}}\right\rangle}$ in the center cell in these different collisions, and we see $T_{\epsilon}>T_{\left\langle p_{\mathrm{T}}\right\rangle}$ in each case. It is also seen that the initial temperature $T_{\epsilon}$ (and $T_{\left\langle p_{\mathrm{T}}\right\rangle}$ ) in mid-central collisions is similar to that in central collisions at the same energy, but the temperature in mid-central collisions decreases faster with time. On the other hand, the initial temperature $T_{\epsilon}\left(\right.$ and $\left.T_{\left\langle p_{\mathrm{T}}\right\rangle}\right)$ in LHC collisions is higher than that in RHIC collisions at the same centrality, but the shape of the temperature evolution with time looks similar at these two energies. 


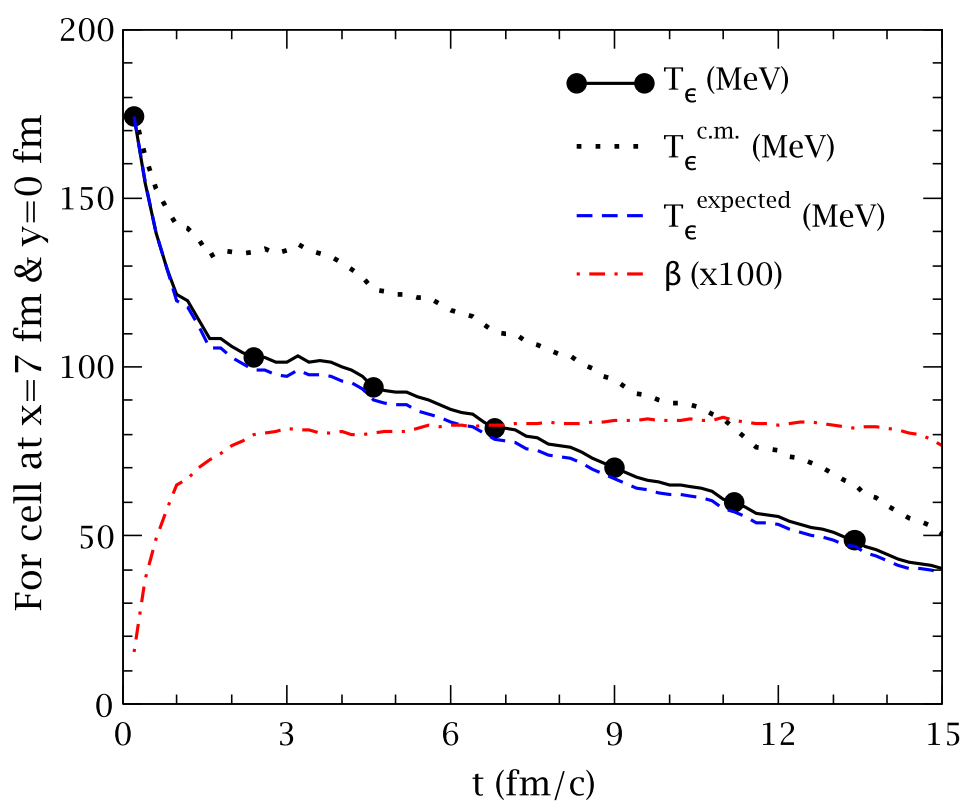

FIG. 8: (Color online) AMPT-SM results on effective temperatures as functions of time for the cell at $x=7 \mathrm{fm} \& y=0 \mathrm{fm}$ in central $\mathrm{Au}+\mathrm{Au}$ collisions at $200 \mathrm{~A} \mathrm{GeV}$; the dot-dashed curve represents the flow magnitude (multiplied by 100) in the cell.

I have also checked the relation between $T_{\epsilon}$ extracted from the parton energy density in the rest frame of the cell and $T_{\epsilon}^{c . m}$., which is the "apparent" temperature if one simply converts the energy density of the cell in the center-of-mass (c.m.) frame into temperature using Eq. (3). Fig. 8 shows the time evolutions of $T_{\epsilon}$ (filled circles) and $T_{\epsilon}^{\text {c.m. }}$ (dotted curve) in the cell at $x=7 \mathrm{fm} \& y=0 \mathrm{fm}$ in central $\mathrm{Au}+\mathrm{Au}$ collisions

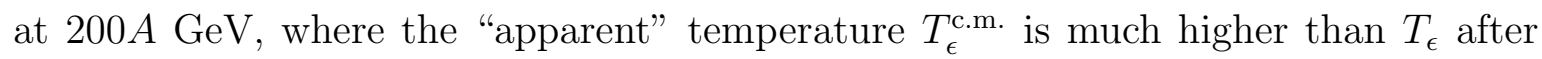
$1 \mathrm{fm} / c$. For a volume cell that can be described by energy density $\epsilon$ and pressure $P$, the energy density $\epsilon$ in its rest frame is related to the energy density $\epsilon^{\text {c.m. }}$ in the c.m. frame by

$$
\epsilon^{\mathrm{c.m.} .}=\gamma^{2}(\epsilon+P)-P
$$

where $\gamma$ is the Lorentz factor for the moving cell in the c.m. frame. Using $P=\epsilon / 3$ for a massless parton gas, I get

$$
\epsilon=\epsilon^{\text {c.m. }}\left(\frac{3}{4 \gamma^{2}-1}\right)
$$




$$
T_{\epsilon}^{\text {expected }}=T_{\epsilon}^{\mathrm{c} . \mathrm{m} .}\left(\frac{1-\beta^{2}}{1+\beta^{2} / 3}\right)^{1 / 4},
$$

where $\beta$ is the magnitude of the flow of the cell in the c.m. frame. Using the flow magnitude $\beta$ shown as the dot-dashed curve in Fig. 8, Eq. (7) gives $T_{\epsilon}^{\text {expected }}$ (the expected value of $T_{\epsilon}$ ) as the dashed curve, which is very close to the actual $T_{\epsilon}$ calculated directly from the energy density in the rest frame of the cell. The small difference between $T_{\epsilon}$ and $T_{\epsilon}^{\text {expected }}$ may come from the fact that partons are not massless and that pressures along different axes are not fully isotropicalized during the evolution [31, 35].

\section{Spatial dependence of effective temperatures}

I now look at how the effective temperatures depend on location in the overlap volume. Fig. 9 shows how $T_{\epsilon}$ and $T_{\left\langle p_{\mathrm{T}}\right\rangle}$ change with location along the impact parameter axis at different times: $t=0.2$ and $2.0 \mathrm{fm} / c$. In both central $\mathrm{Pb}+\mathrm{Pb}$ collisions at $2760 A$ $\mathrm{GeV}$ and mid-central $\mathrm{Au}+\mathrm{Au}$ collisions at $200 \mathrm{~A} \mathrm{GeV}$, we see that the initial effective temperatures $T_{\left\langle p_{\mathrm{T}}\right\rangle}$ at $t=0.2 \mathrm{fm} / c$ change little over most of the overlap volume. We

also see that $T_{\epsilon}>T_{\left\langle p_{\mathrm{T}}\right\rangle}$ over the inner part of the overlap volume, e.g., within $|x|<6$ fm for central $\mathrm{Pb}+\mathrm{Pb}$ collisions at $2760 A \mathrm{GeV}$ and within $|x|<3 \mathrm{fm}$ for mid-central $\mathrm{Au}+\mathrm{Au}$ collisions at $200 \mathrm{~A} \mathrm{GeV}$ at $t=0.2 \mathrm{fm} / c$. Note that, in order to limit the statistical fluctuations, volume cells that have less than 20 partons after summing over simulated events are not shown in Figs. $9,10$.

The spatial dependences of effective temperatures $T_{\epsilon}$ and $T_{\left\langle p_{\mathrm{T}}\right\rangle}$ in three different collision systems are shown in Fig. 10a for $t=0.2 \mathrm{fm} / c$ and in Fig. 10p for $t=5.0$ fm/c. From Fig. 10a I find that the initial effective temperatures $T_{\left\langle p_{\mathrm{T}}\right\rangle}$ for central and mid-central $\mathrm{Pb}+\mathrm{Pb}$ collisions at $2760 \mathrm{~A} \mathrm{GeV}$ are almost the same in the overlap volume except that the spatial width is smaller for mid-central collisions. In contrast, the initial effective temperatures $T_{\epsilon}$ for central LHC collisions are higher and much wider in spatial width than those in mid-central LHC collisions. These features are also seen in results at the RHIC energy. Furthermore, although the initial temperatures $T_{\epsilon}$ in mid-central $\mathrm{Pb}+\mathrm{Pb}$ collisions at $2760 \mathrm{~A} \mathrm{GeV}$ are higher than those in central $\mathrm{Au}+\mathrm{Au}$ collisions at 


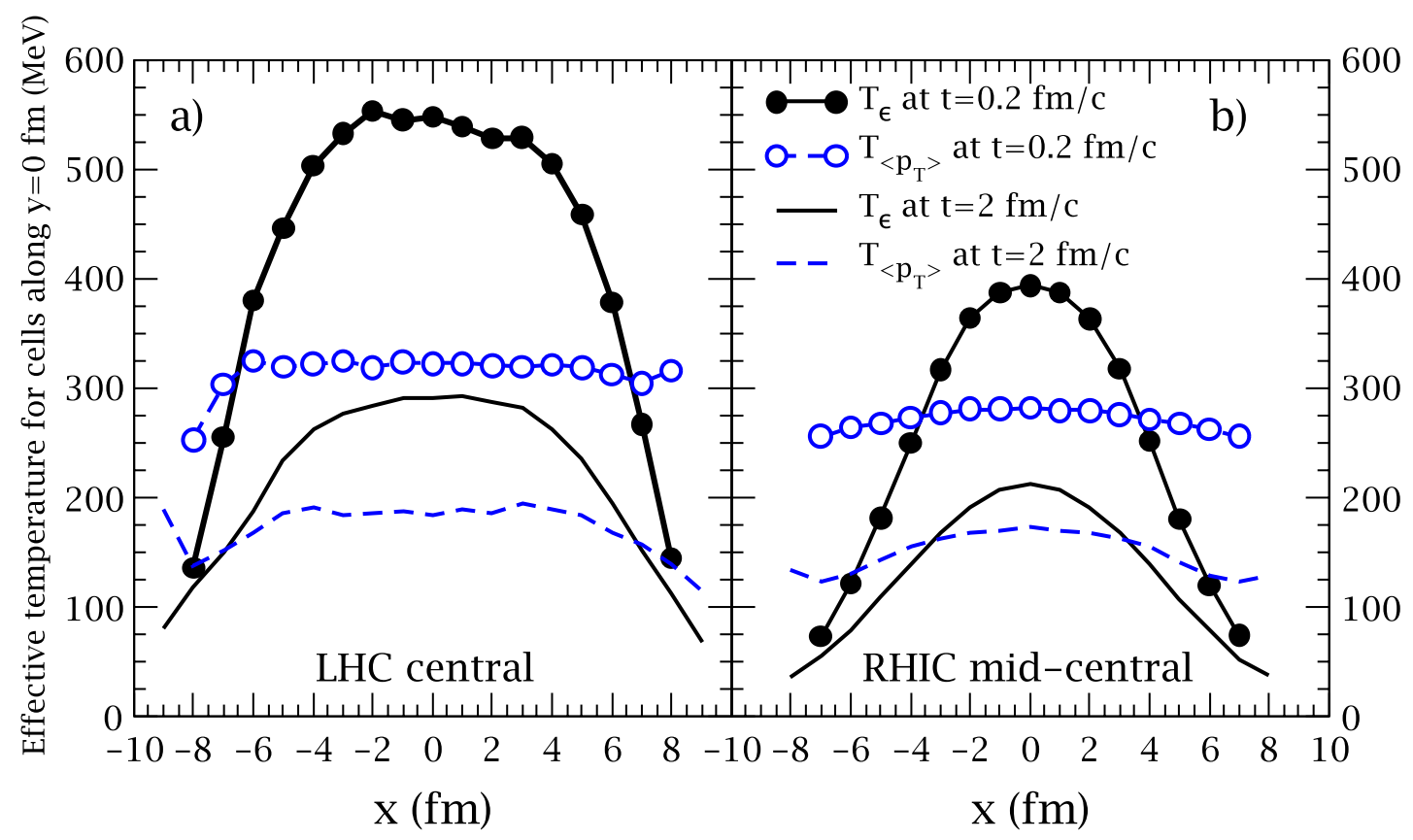

FIG. 9: (Color online) AMPT-SM results on effective temperatures in cells along the impact parameter at $t=0.2$ and $2.0 \mathrm{fm} / c$ : a) for central $\mathrm{Pb}+\mathrm{Pb}$ collisions at $2760 \mathrm{~A} \mathrm{GeV}$, and b) for mid-central $\mathrm{Au}+\mathrm{Au}$ collisions at $200 \mathrm{~A} \mathrm{GeV}$.

$200 \mathrm{~A} \mathrm{GeV}$ in the inner part of the overlap volume, the width of $T_{\epsilon}$ along the impact parameter axis is smaller. From Fig. 10p one sees that the effective temperature $T_{\epsilon}$ at $t=5.0 \mathrm{fm} / c$ for central LHC collisions are overall significantly higher than the other collisions systems. In addition, temperatures $T_{\epsilon}\left(\right.$ and $\left.T_{\left\langle p_{\mathrm{T}}\right\rangle}\right)$ at $t=5.0 \mathrm{fm} / c$ are mostly similar for mid-central $\mathrm{Pb}+\mathrm{Pb}$ collisions at $2760 A \mathrm{GeV}$ and central $\mathrm{Au}+\mathrm{Au}$ collisions at $200 \mathrm{~A} \mathrm{GeV}$, suggesting that the lifetime of the quark-gluon plasma is similar in these two collision systems.

\section{Over-population of partons}

We have already seen from Figs. 9,10 that $T_{\epsilon}>T_{\left\langle p_{\mathrm{T}}\right\rangle}$ over the inner part of the overlap volume in these high energy heavy ion collisions, and this is essentially due to $T_{n}>T_{\left\langle p_{\mathrm{T}}\right\rangle}$ according to Eq. (5). This reflects the fact that the parton system in a subvolume of the overlap region in such a collision, even after being averaged 


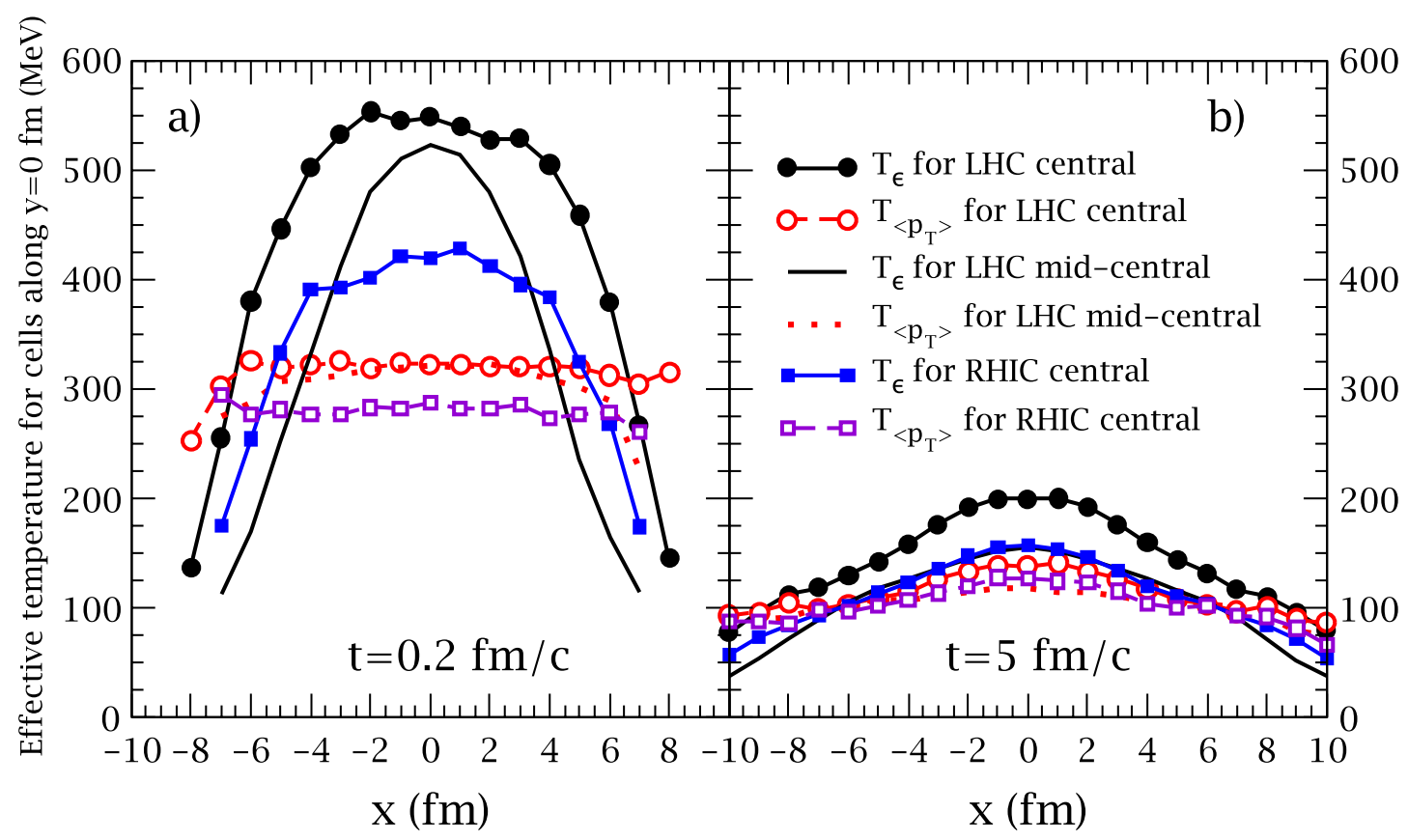

FIG. 10: (Color online) AMPT-SM results on effective temperatures in cells along the impact parameter for different collisions: a) at $t=0.2 \mathrm{fm} / c$, and b) at $t=5.0 \mathrm{fm} / c$.

over many events at the same impact parameter, is not in full chemical equilibrium as defined for an ideal quark-gluon plasma. The relation $T_{n}>T_{\left\langle p_{\mathrm{T}}\right\rangle}$ means that for the parton number density we have $n>n\left(T_{\left\langle p_{\mathrm{T}}\right\rangle}\right)$, where $n\left(T_{\left\langle p_{\mathrm{T}}\right\rangle}\right)$ is the parton number density expected for a quark-gluon plasma in full chemical equilibrium at temperature $T_{\left\langle p_{\mathrm{T}}\right\rangle}$. For example, $T_{\epsilon} \sim 1.7 T_{\left\langle p_{\mathrm{T}}\right\rangle}$ in Figs. $9 \mathrm{a}$ for $x=0 \mathrm{fm}$ at $t=0.2 \mathrm{fm} / \mathrm{c}$ in central LHC collisions means that, according to Eq. (3) and the approximation of Eq. (5), $n \sim 1.7^{4} n\left(T_{\left\langle p_{\mathrm{T}}\right\rangle}\right) \simeq 8.4 n\left(T_{\left\langle p_{\mathrm{T}}\right\rangle}\right)$ for that center cell at that time. Later at $t=5.0$ $\mathrm{fm} / c$, we see in Figs. 9b that $T_{\epsilon} \sim 1.44 T_{\left\langle p_{\mathrm{T}}\right\rangle}$, which gives $n \sim 4.3 n\left(T_{\left\langle p_{\mathrm{T}}\right\rangle}\right)$ at that time. Therefore one may say that the parton system in that subvolume is over-populated, in that the parton density is too high compared to that expected for an ideal QGP that has the same parton mean transverse momentum.

To further illustrate this, I calculate the phase-space density of partons. In terms of the magnitude of parton 3-momentum in the rest frame of a cell, I have

$$
f(p) \equiv(2 \pi)^{3} \frac{d N}{d^{3} x d^{3} p}=2 \pi^{2} \frac{d N / d^{3} x}{p^{2} d p} .
$$




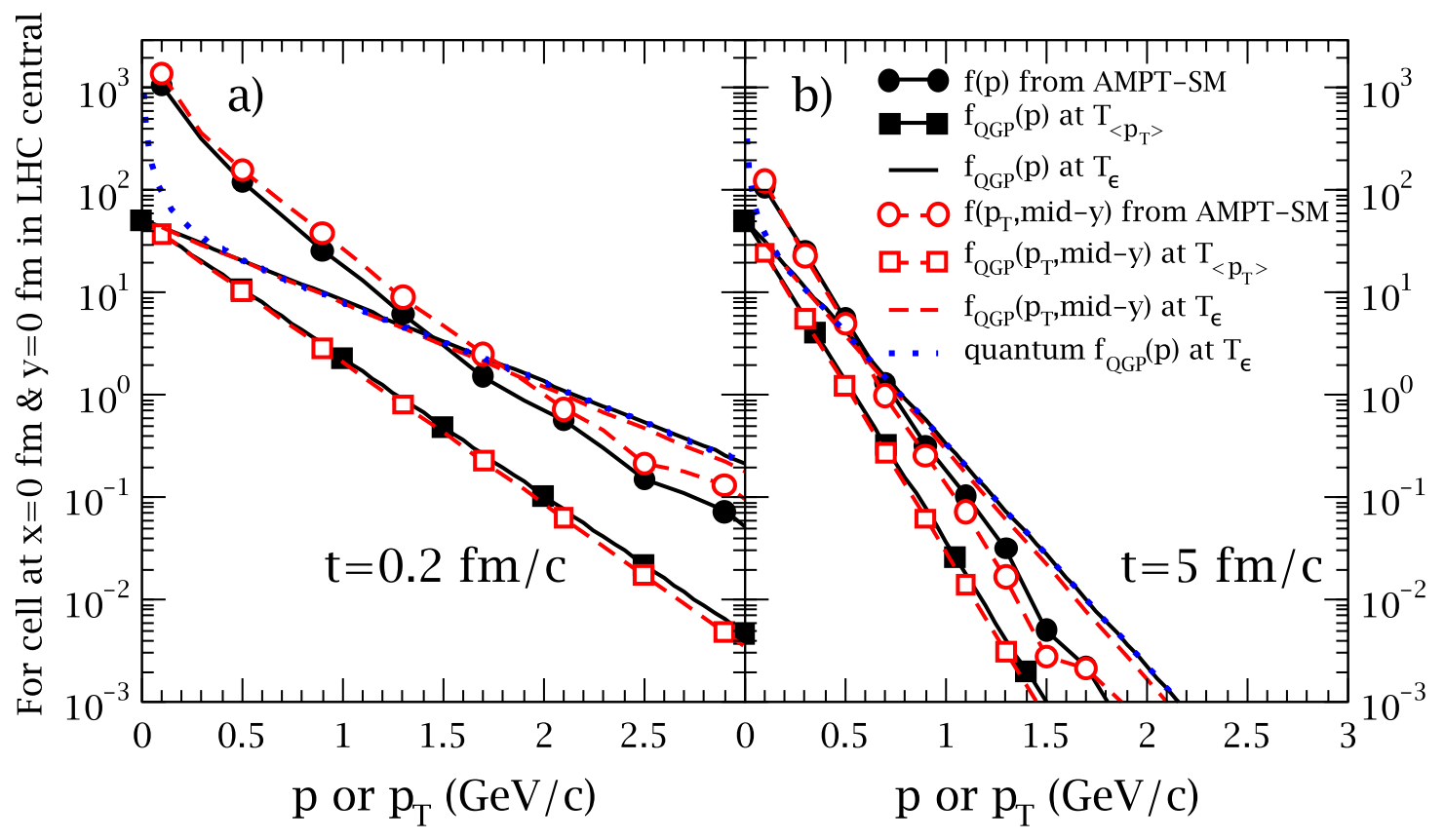

FIG. 11: (Color online) Phase-space distribution of partons in the center cell in central $\mathrm{Pb}+\mathrm{Pb}$ collisions at $2760 A \mathrm{GeV}$ versus momentum $p$ (solid curves) or versus $p_{\mathrm{T}}$ at midrapidity (dashed curves) a) at $t=0.2 \mathrm{fm} / c$, and b) at $t=5.0 \mathrm{fm} / c$. Circles represent results from the AMPT-SM model, curves with squares represent an ideal QGP at temperature $T_{\left\langle p_{\mathrm{T}}\right\rangle}$, solid and dashed curves without symbols represent an ideal QGP at temperature $T_{\epsilon}$, and dotted curves represent an ideal QGP at temperature $T_{\epsilon}$ with quantum statistics.

I also calculate the phase-space density in terms of parton $p_{\mathrm{T}}$ at a given rapidity $y$ in the rest frame of a cell, where one can write

$$
f\left(p_{\mathrm{T}}, y\right)=4 \pi^{2} \frac{d N / d^{3} x}{p_{\mathrm{T}} d p_{\mathrm{T}} d p_{\mathrm{z}}}
$$

I choose mid-rapidity partons, i.e., partons within $|y|<1 / 2$ in the rest frame of the cell. By approximating the parton system as massless, which is a reasonable approximation when the temperature is high (see left panel of Fig. 6), I obtain

$$
f\left(p_{\mathrm{T}}, \operatorname{mid}-\mathrm{y}\right) \simeq 4 \pi^{2} \frac{d N / d^{3} x}{p_{\mathrm{T}} d p_{\mathrm{T}} \Delta p_{\mathrm{z}}} \simeq \frac{4 \pi^{2}}{2 \sinh (1 / 2)} \frac{d N / d^{3} x}{p_{\mathrm{T}}^{2} d p_{\mathrm{T}}},
$$

where $\Delta p_{\mathrm{z}}$ represents the longitudinal momentum range under consideration. For comparison, the expected phase-space distribution for an ideal QGP at temperature $T$ is 
given by

$$
f_{\mathrm{QGP}}(p)=g_{\mathrm{B}} e^{-E / T} \simeq g_{\mathrm{B}} e^{-p / T}, f_{\mathrm{QGP}}\left(p_{\mathrm{T}}, y\right)=g_{\mathrm{B}} e^{-m_{\mathrm{T}} \cosh (y) / T}
$$

when the Boltzmann distribution is used for partons that are assumed to be massless. I then make the following approximation for mid-rapidity:

$$
f_{\mathrm{QGP}}\left(p_{\mathrm{T}}, \operatorname{mid}-\mathrm{y}\right) \simeq g_{\mathrm{B}} e^{-p_{\mathrm{T}} \cosh (1 / 4) / T}
$$

The AMPT-SM results for the center cell at two different times in central $\mathrm{Pb}+\mathrm{Pb}$ collisions at $2760 A \mathrm{GeV}$ are shown in Fig. 11, in comparison with the expected phasespace distributions for an ideal QGP at the corresponding temperature $T_{\epsilon}(548 \mathrm{MeV}$ at $t=0.2 \mathrm{fm} / c$ and $199 \mathrm{MeV}$ at $t=5 \mathrm{fm} / c)$ or $T_{\left\langle p_{\mathrm{T}}\right\rangle}(322 \mathrm{MeV}$ at $t=0.2 \mathrm{fm} / c$ and $138 \mathrm{MeV}$ at $t=5 \mathrm{fm} / c$ ). We first see that the AMPT-SM results (curves with circles) and the expected curves for an ideal QGP at temperature $T_{\left\langle p_{\mathrm{T}}\right\rangle}$ (curves with squares) at a given time have a similar overall slope. This reflects the fact that the effective temperature $T_{\left\langle p_{\mathrm{T}}\right\rangle}$ is only determined by the parton mean $p_{\mathrm{T}}$ in the cell; it also indicates that the $p_{\mathrm{T}}$ distribution of partons in the subvolume here is not too far from a thermal distribution. In the meantime, the AMPT-SM curves are much higher in magnitude than the ideal QGP curves at temperature $T_{\left\langle p_{\mathrm{T}}\right\rangle}$, suggesting that the parton matter is over-populated by a large factor. When compared with the expected distributions for an ideal QGP at temperature $T_{\epsilon}$ (solid and dashed curves without symbols), partons in the AMPT-SM results are still over-populated below a given value of $p_{\mathrm{T}}$ or $p$. This value is smaller at a later time as shown in Fig. 11: partons in that center cell are seen to be over-populated below $p_{\mathrm{T}} \simeq 1.4 \mathrm{GeV} / c$ at $t=0.2 \mathrm{fm} / c$ but below $p_{\mathrm{T}} \simeq 0.6 \mathrm{GeV} / c$ at $t=5 \mathrm{fm} / c$, when compared with an ideal QGP at temperature $T_{\epsilon}$.

From Fig. 11 we see that the phase-space distributions in momentum $p$ or $p_{\mathrm{T}}$ are quite close to each other. At zero-momentum, both $f_{\mathrm{QGP}}(p)$ and $f_{\mathrm{QGP}}\left(p_{\mathrm{T}}, \mathrm{mid}-\mathrm{y}\right)$ approach the value $g_{\mathrm{B}}=52$ (the degeneracy factor of QGP for $N_{f}=3$ ) for an ideal QGP with Boltzmann statistics. Considering Fermi-Dirac statistics, the phase-space densities of quarks and anti-quarks are bound to be below one for each degree of freedom due to the Pauli exclusion principle, therefore the phase space density contributed from quarks and anti-quarks must be below $12 N_{f}=36$ (their total degeneracy factor for 
$\left.N_{f}=3\right)$ regardless of temperature. Therefore the high phase-space densities above this value shown in Fig. 11 cannot be explained by a full population of the phase space by quarks and antiquarks. It cannot be explained by the Bose-Einstein distribution of gluons in an ideal QGP either. Dotted curves in Fig. 11 represent the expected quantum phase-space distributions at temperature $T_{\epsilon}$ when Bose-Einstein and FermiDirac distributions are used for a massless quark-gluon plasma:

$$
f_{\mathrm{QGP}}^{q u a n t u m}(p)=\frac{16}{e^{p / T}-1}+\frac{12 N_{f}}{e^{p / T}+1} .
$$

For an ideal QGP, we see that the expected quantum phase-space densities are very close to the expected Boltzmann densities except at very low $p_{\mathrm{T}}$, and the AMPTSM results are still much higher than the quantum phase-space densities except at extremely low $p_{\mathrm{T}}$. Since there is no bound for the gluon phase-space densities due to quantum statistics, one may argue that the high density regions as shown in Fig. 11 indicate that at least gluons are over-populated there. This may be analogous to the glasma produced from the color-glass-condensate [2, 36].

Therefore, when the parton system in a volume cell satisfies $n>n\left(T_{\left\langle p_{\mathrm{T}}\right\rangle}\right)$, I consider partons in that cell as being over-populated relative to an ideal QGP at temperature $T_{\left\langle p_{\mathrm{T}}\right\rangle}$. So I use the AMPT-SM results to investigate regions within $|\eta|<1 / 2$ that have over-populated partons. I first define a "critical" temperature $T_{c}$ above which a dense matter can be considered as being well inside the QGP phase, then a cell that satisfies the condition $T_{\epsilon}>T_{c}$ is called a QGP cell, and a QGP cell that also satisfies the condition $T_{\epsilon}>T_{\left\langle p_{\mathrm{T}}\right\rangle}$ is called an over-populated cell. In this study I take $T_{c}=150 \mathrm{MeV}$, which corresponds to a "critical" energy density $\epsilon_{c}=1.05 \mathrm{GeV} / \mathrm{fm}^{3}$ for $N_{f}=3$ as given by Eq. (3).

Fig. 12 shows the transverse locations of QGP cells (i.e. with $T_{\epsilon}>T_{c}$ ) and overpopulated cells (i.e. with $T_{\epsilon}>T_{c}$ and $T_{\epsilon}>T_{\left\langle p_{\mathrm{T}}\right\rangle}$ ) in mid-central $\mathrm{Pb}+\mathrm{Pb}$ collisions at $2760 \mathrm{~A} \mathrm{GeV}$. We can see that at $t=0.2 \mathrm{fm} / c$ the initial extension of QGP cells (filled circles) along the $y$-axis is bigger than that along the $x$-axis due to the spatial asymmetry in mid-central collisions. More that half of these QGP cells are overpopulated at this early time, as indicated by filled squares, and they are located in the inner part of the overlap volume. Later at $t=3.0 \mathrm{fm} / c$, QGP cells that are over- 


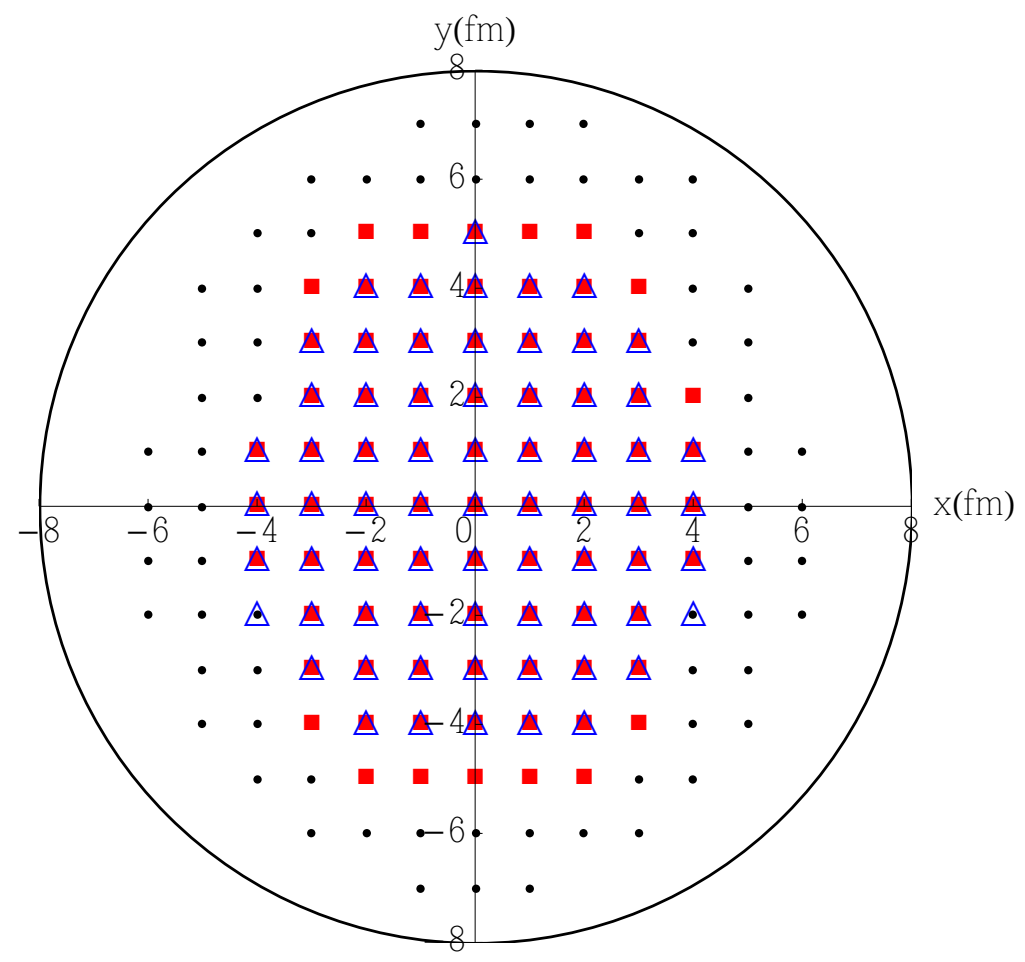

FIG. 12: (Color online) For partons within $|\eta|<1 / 2$ in mid-central $\mathrm{Pb}+\mathrm{Pb}$ collisions at $2760 \mathrm{~A} \mathrm{GeV}$, filled circles represent the center locations in the transverse plane for QGP cells at $t=0.2 \mathrm{fm} / c$, filled squares represent over-populated QGP cells at $t=0.2 \mathrm{fm} / c$, and open triangles represent over-populated QGP cells at $t=3.0 \mathrm{fm} / c$. The circle is drawn as a reference shape.

populated (open triangles) still occupy an area in the transverse plane almost as large as that at $t=0.2 \mathrm{fm} / c$ (filled squares), while the shape of overpopulated cells in the transverse plane at $t=3.0 \mathrm{fm} / c$ has a smaller spatial asymmetry than that at $t=0.2$ $\mathrm{fm} / c$.

In Fig. 13 I show the transverse areas of QGP cells and the transverse areas of over-populated QGP cells as functions of time in different collisions. Note that each cell here covers $|\eta|<1 / 2$ along the beam direction. For all four collision systems, we see that initially a significant fraction, roughly from $50 \%$ to $70 \%$, of the QGP cells are over-populated with partons. This fraction is higher for central collisions than mid-central collision at the same energy and also higher for the LHC energy than the RHIC energy at the same centrality. After $2-3 \mathrm{fm} / c$, however, all QGP cells are over- 


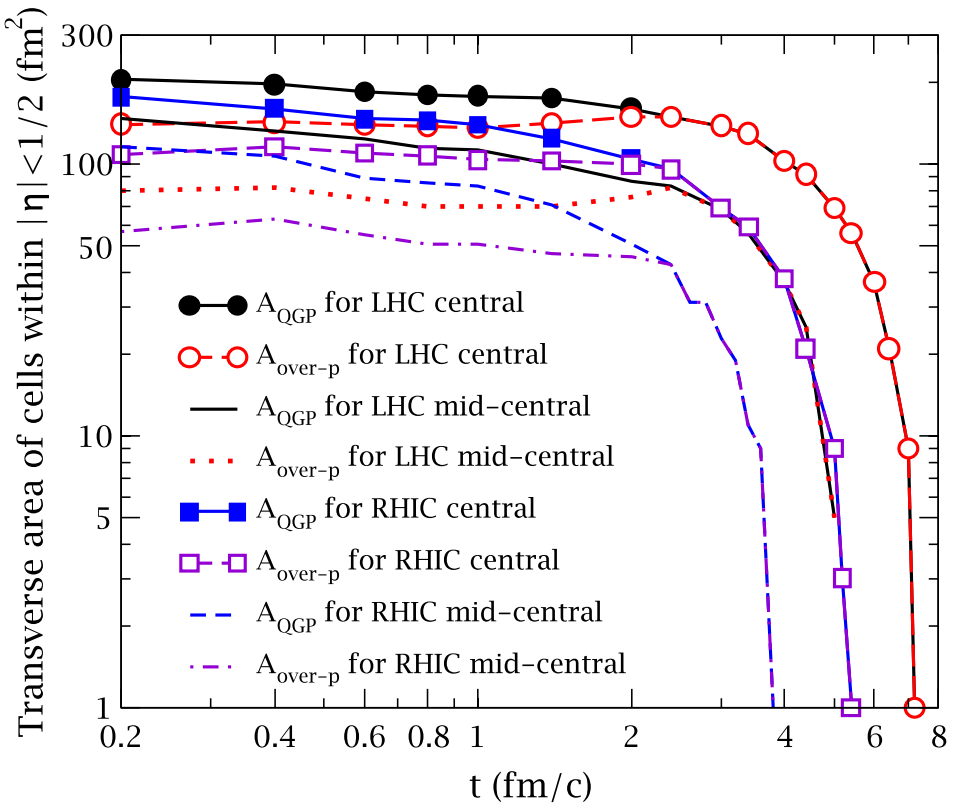

FIG. 13: (Color online) Transverse areas of QGP cells $\left(A_{\mathrm{QGP}}\right)$ and transverse areas of QGP cells that are over-populated $\left(A_{\text {over-p }}\right)$ as functions of time for different collisions.

populated. Although the transverse area of QGP cells decreases monotonously over time, the transverse area of over-populated cells does not change much and may even slightly increase over the first several $\mathrm{fm} / \mathrm{c}$ of time. One also sees that the areas in central RHIC collisions are mostly bigger than the corresponding areas in mid-central LHC collisions even though the peak temperature in central RHIC collisions is lower (as shown in Fig. 10a), while the QGP phases in the two collision systems show a similar lifetime.

\section{E. Describing parton over-population with parton phase-space occupancy}

\section{factors}

We can represent the over-population of partons with parton phase-space occupancy factors. Let us introduce the following quantum phase-space distribution function for QGP that has a zero baryon chemical potential but is off chemical equilibrium:

$$
f_{\mathrm{QGP}}^{n o n-e q}(p)=\frac{16 \gamma_{g}}{e^{p / T}-1}+\frac{12 N_{f} \gamma_{q}}{e^{p / T}+1}
$$


where $\gamma_{g}$ is the gluon phase-space occupancy and $\gamma_{q}$ is the quark (and anti-quark) phase-space occupancy. The above distribution gives the energy density as $\epsilon^{n o n-e q}(T)=$ $\pi^{2}\left(16 \gamma_{g}+10.5 N_{f} \gamma_{q}\right) T^{4} / 30$. Since the effective temperature $T_{\left\langle p_{\mathrm{T}}\right\rangle}$ represents the shape of the phase-space distribution, it will be used as temperature $T$ in Eq. (14), and matching $\epsilon^{n o n-e q}\left(T_{\left\langle p_{\mathrm{T}}\right\rangle}\right)$ to the local energy density value $\epsilon \equiv 3 g_{\mathrm{B}} T_{\epsilon}^{4} / \pi^{2}$ enables us to extract the gluon phase-space occupancy of each volume cell as

$$
\gamma_{g}=\frac{3}{32}\left[\frac{240\left(4+3 N_{f}\right)}{\pi^{4}}\left(\frac{T_{\epsilon}}{T_{\left\langle p_{\mathrm{T}}\right\rangle}}\right)^{4}-7 N_{f} \gamma_{q}\right] .
$$

Alternatively we can use the following Boltzmann distribution function for a massless QGP that is off chemical equilibrium:

$$
f_{\mathrm{QGP}, \mathrm{B}}^{n o n-e q}(p)=16 \gamma_{g} e^{-p / T}+12 N_{f} \gamma_{q} e^{-p / T},
$$

which leads to the energy density as $\epsilon_{B}^{n o n-e q}(T)=12\left(4 \gamma_{g}+3 N_{f} \gamma_{q}\right) T^{4} / \pi^{2}$. Matching $\epsilon_{B}^{n o n-e q}\left(T_{\left\langle p_{\mathrm{T}}\right\rangle}\right)$ to the local energy density value $\epsilon \equiv 3 g_{\mathrm{B}} T_{\epsilon}^{4} / \pi^{2}$ then yields the gluon phase-space occupancy for Boltzmann distributions as

$$
\gamma_{g}=\frac{1}{4}\left[\left(4+3 N_{f}\right)\left(\frac{T_{\epsilon}}{T_{\left\langle p_{\mathrm{T}}\right\rangle}}\right)^{4}-3 N_{f} \gamma_{q}\right] .
$$

As the gluon and quark composition of the partonic matter cannot be addressed currently via the AMPT-SM model, the relationship between $\gamma_{g}$ and $\gamma_{q}$ is unknown. However, since $\gamma_{q} \in[0,1]$ due to the Pauli exclusion principle, we can obtain the range of $\gamma_{g}$ as $\left[\gamma_{g}^{\min }, \gamma_{g}^{\max }\right]$, where $\gamma_{g}^{\min }$ is the value of $\gamma_{g}$ for $\gamma_{q}=1$ and $\gamma_{g}^{\max }$ is the value of $\gamma_{g}$ for $\gamma_{q}=0$.

Fig. 14 shows the phase-space distributions of partons in the center cell of central $\mathrm{Pb}+\mathrm{Pb}$ collisions at $2760 \mathrm{~A} \mathrm{GeV}$ from the AMPT-SM results at two different times (curves with circles). Also shown for comparisons are the quantum phase-space distributions of Eq. (14) (solid curves and dashed curves) and the Boltzmann distribution of Eq. (16) (dotted curves) for QGP in non-chemical-equilibrium at the corresponding temperature $T_{\left\langle p_{\mathrm{T}}\right\rangle}(322 \mathrm{MeV}$ at $t=0.2 \mathrm{fm} / c$ and $138 \mathrm{MeV}$ at $t=5 \mathrm{fm} / c)$. We see that all three non-chemical-equilibrium distributions in Fig. 14 describe the AMPT-SM results much better than the chemical-equilibrium phase-space distributions shown in Fig. 11. We also see that the non-equilibrium quantum phase-space 


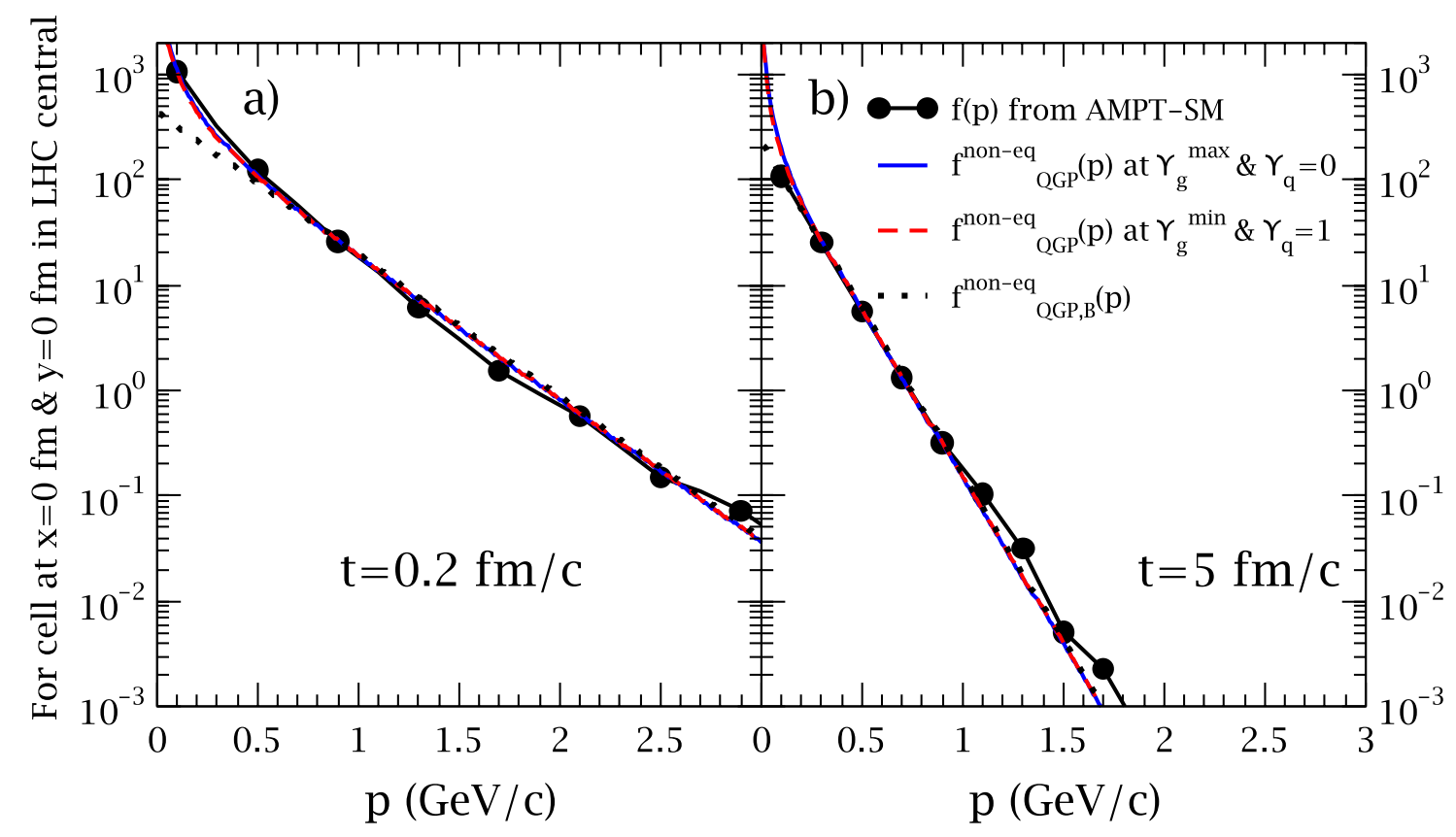

FIG. 14: (Color online) Phase-space distribution of partons in the center cell in central $\mathrm{Pb}+\mathrm{Pb}$ collisions at $2760 A \mathrm{GeV}$ versus momentum $p$ from AMPT-SM a) at $t=0.2 \mathrm{fm} / c$, and b) at $t=5.0 \mathrm{fm} / c$, in comparison with the corresponding non-equilibrium quantum distributions of Eq. 14 at $\gamma_{g}=\gamma_{g}^{\max } \& \gamma_{q}=0$ and at $\gamma_{g}=\gamma_{g}^{\min } \& \gamma_{q}=1$ and the non-equilibrium Boltzmann distribution of Eq. (16).

distributions for two sets of parton phase-space occupancies, $\left(\gamma_{g}=\gamma_{g}^{\max }, \gamma_{q}=0\right)$ and $\left(\gamma_{g}=\gamma_{g}^{\min }, \gamma_{q}=1\right)$, are essentially the same; while the range of $\gamma_{g}$ for the non-equilibrium quantum distribution is $[23.1,25.1]$ for Fig. 14a and [11.1, 13.1] for Fig. 14b. Since the Boltzmann distribution does not distinguish between bosons and fermions, the non-equilibrium Boltzmann phase-space distribution $f_{\mathrm{QGP}, \mathrm{B}}^{n o n-e q}(p)$ (dotted curves) is exactly the same for phase-space occupancies $\left(\gamma_{g}=\gamma_{g}^{\max }, \gamma_{q}=0\right)$ and $\left(\gamma_{g}=\gamma_{g}^{\min }, \gamma_{q}=1\right)$; while the range of $\gamma_{g}$ for the non-equilibrium Boltzmann distribution is $[24.9,27.2]$ for Fig. 14 and $[11.9,14.2]$ for Fig. 14 b. We see that the gluon phase-space occupancy can be much bigger than one. In addition, using the nonequilibrium quantum or Boltzmann distribution does not affect the extracted range of $\gamma_{g}$ too much. 


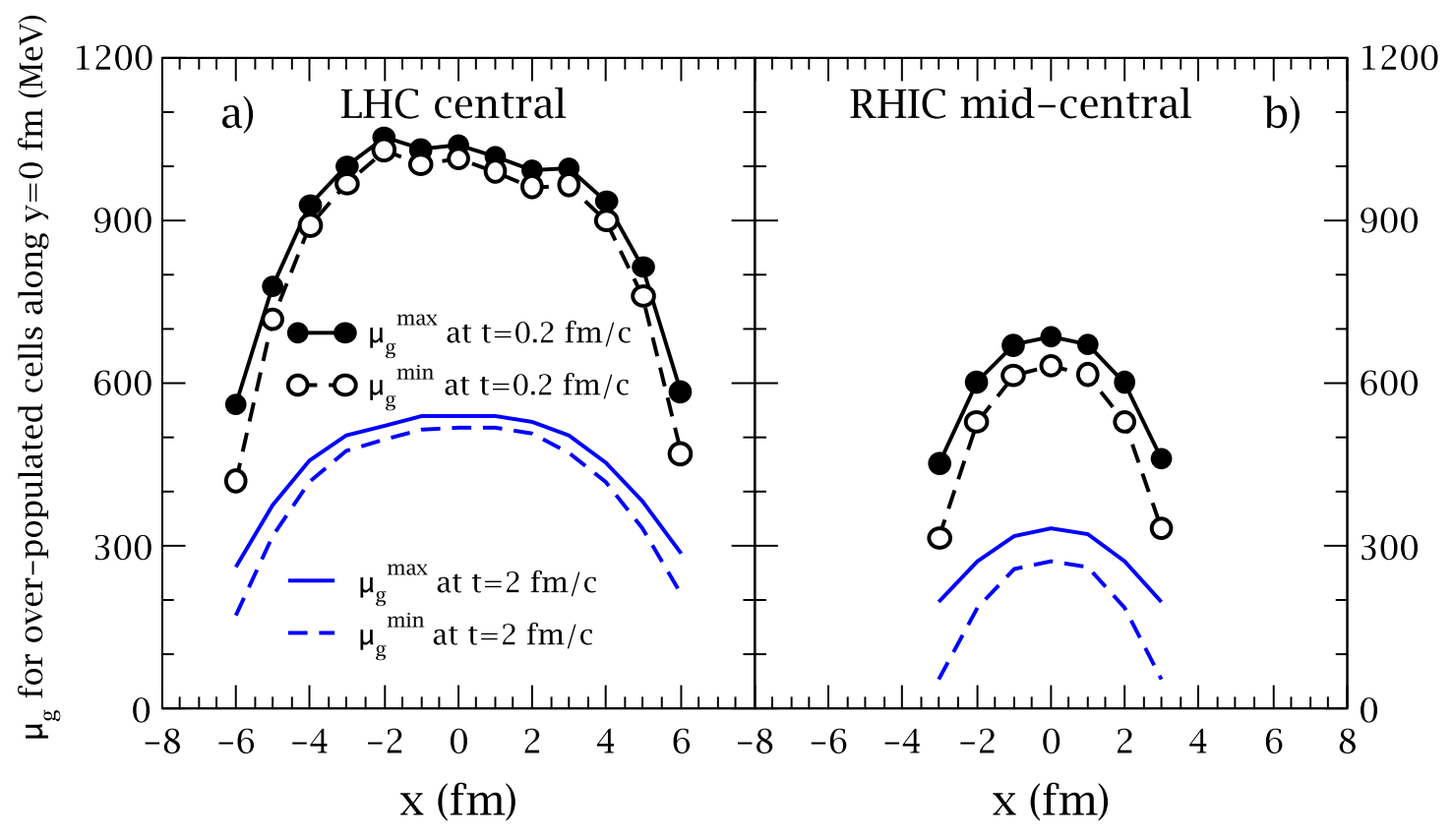

FIG. 15: (Color online) Extracted range of $\mu_{g}$, the gluon "chemical potential" parameter, in over-populated cells along the impact parameter at $t=0.2$ and $2.0 \mathrm{fm} / c$ : a) for central $\mathrm{Pb}+\mathrm{Pb}$ collisions at $2760 A \mathrm{GeV}$, and b) for mid-central $\mathrm{Au}+\mathrm{Au}$ collisions at $200 \mathrm{~A} \mathrm{GeV}$.

The gluon phase-space occupancy $\gamma_{g}$ may be conveniently translated into the gluon "chemical potential" parameter $\mu_{g}$ via the definition

$$
\gamma_{g} \equiv e^{\left(\mu_{g} / T_{\left.<p_{\mathrm{T}}>\right)}\right.}
$$

I can then obtain the range of $\mu_{g}$ as $\left[\mu_{g}^{\min }, \mu_{g}^{\max }\right]$ that corresponds to the $\gamma_{g}$ range $\left[\gamma_{g}^{\min }, \gamma_{g}^{\max }\right]$. Fig. 15 shows the extracted range of $\mu_{g}$, which corresponds to the gluon phase-space occupancy $\gamma_{g}$ of Eq. (15) for the non-equilibrium quantum distribution, in over-populated cells along the impact parameter axis at two different times for central $\mathrm{Pb}+\mathrm{Pb}$ collisions at $2760 A \mathrm{GeV}$ and mid-central $\mathrm{Au}+\mathrm{Au}$ collisions at $200 \mathrm{~A} \mathrm{GeV}$. Although the quark and anti-quark phase-space occupancy $\gamma_{q}$ cannot be determined, we see that this does not lead to big uncertainties in the extracted gluon "chemical potential" parameter $\mu_{g}$. We also see that the gluon "chemical potential" is larger in the inner part of the overlap volume and can reach $1 \mathrm{GeV}$ in the very early stage of central $\mathrm{Pb}+\mathrm{Pb}$ collisions at LHC. 


\section{DISCUSSIONS}

The string melting version [10, 15] of the AMPT model only has quarks and antiquarks in the parton phase. This is not to be considered as physical though, since one expects gluons to dominate the initial stage of ultra-relativistic heavy ion collisions and gluons will also be produced from quark and anti-quark interactions. The lack of gluons in the AMPT-SM model results from our inability to consistently address gluon and quark productions from a strong color field [10]. However, the AMPT-SM model enables us to include the energy from all the excited strings in the overlap volume into the parton transport. For studies that depend more on the effect of partonic scatterings instead of the composition of the partonic matter, such as studies of the elliptic flow [15] and the pion interferometry [37, it is suitable to use the AMPT-SM model. Since this study addresses the time evolution of parton flow, mean momentum or energy, number density, and energy density, its results depend mostly on the effect of partonic scatterings but not on the composition of the partonic matter. I then infer

from the effective temperatures $T_{\epsilon}$ and $T_{\left\langle p_{\mathrm{T}}\right\rangle}$, which are extracted respectively from the parton energy density and mean $p_{\mathrm{T}}$, that the high density matter in the inner region of the overlap volume in these collisions are over-populated with partons (at least over-populated with gluons), even though the AMPT-SM model has no gluons in the parton phase. However, note that further studies, such as a consistent description of the initial production of gluons and quarks from strong color fields, will be needed to investigate whether this phase-space over-population is a general feature [2, 38] of the QCD matter created in high energy heavy ion collisions. Currently I may conclude that this is the feature of the parton system within the string melting version of our microscopic transport model, which has been constrained to reproduce the bulk data on the rapidity density, $p_{\mathrm{T}}$ spectrum and elliptic flow at low $p_{\mathrm{T}}$.

The parton cascade in the AMPT model only includes elastic parton scatterings. Including inelastic parton scatterings in the parton cascade [9, 35] would change the space-time evolution of the dense parton matter and thus affect the transverse flow and effective temperatures. However, since the initial condition from the AMPT-SM model would not be affected by the subsequent parton scatterings, the results in this study at 
$t=0.2 \mathrm{fm} / c$ would remain the same. Therefore the qualitative conclusions about the mismatch among different effective temperatures and about the over-population in the inner part of the overlap volume would not change; while the time evolutions of the temperature mismatch and over-populated areas would be affected by inelastic parton scatterings.

The space-time evolution data obtained from this study may serve as a bulk matter background for studies such as jet propagation and interactions with the partonic matter within the JET Collaboration [23, 39] and beyond. The effective temperatures extracted from the parton phase in the AMPT-SM model also provide a link to hydrodynamic models. Currently one may argue that $T_{\epsilon}$ is a reasonable choice for the effective temperature of the parton system, because it takes into account both the density and the mean momentum of the local parton system. Also, the essential variable in the hydrodynamic approach is the local energy-momentum tensor, and using $T_{\epsilon}$ ensures that the local energy distributions remain the same when the transport approach is linked to the hydrodynamic approach. However, since the effective temperatures depend on the variables from which they are extracted, further studies will be necessary to address the uncertainty associated with this dependence. As we have seen, the mismatch between $T_{\epsilon}$ and $T_{\left\langle p_{\mathrm{T}}\right\rangle}$ could be attributed to an over-population of partons in the dense matter, thus the uncertainty about the effective temperatures reflects the uncertainty in the initial conditions for ultra-relativistic heavy ion collisions.

\section{CONCLUSIONS}

I have studied the space-time evolution of the parton phase created in heavy ion collisions at RHIC and LHC energies using the AMPT model with string melting, which converts excited hadronic strings in the overlap volume into partons. Several key parameters in the model have been tuned to reproduce the low- $p_{\mathrm{T}}$ data on the pion and kaon yields, $p_{\mathrm{T}}$ spectra and elliptic flows. This way the space-time evolution from the model is more reliable, and it may serve as a bulk matter background for studies such as jet propagation and interactions with the partonic matter.

This study focuses on the effective temperatures in different volume cells within 
mid-spacetime-rapidity $|\eta|<1 / 2$. Many events at the same impact parameter are averaged over in order to have enough statistics for the analysis of each volume cell, and as a result this study does not address the effect of event-by-event fluctuations. Since the parton system in a subvolume is in general neither in full thermal equilibrium nor in full chemical equilibrium, the value of the effective temperature depends, sometimes strongly, on the variable that it is extracted from. I have extracted effective temperatures from several different variables that are evaluated in the rest frame of each cell. I find that temperature $T_{\epsilon}$ extracted from the parton energy density is often very different from $T_{\left\langle p_{\mathrm{T}}\right\rangle}$ extracted from the parton mean $p_{\mathrm{T}}$, and it is mostly between $T_{\left\langle p_{\mathrm{T}}\right\rangle}$ and $T_{n}$ extracted from the parton number density while being closer to $T_{n}$. For these collisions I also find that $T_{\epsilon}>T_{\left\langle p_{\mathrm{T}}\right\rangle}$ over the inner part of the overlap volume, which indicates that partons (at least gluons) are over-populated there. This is also checked by examinations of parton phase-space densities, parton phase-space occupancy factors, and the gluon "chemical potential" parameter. My results show that at mid-spacetime-rapidity initially about half or more QGP cells, defined as cells that are above a critical energy density of $1 \mathrm{GeV} / \mathrm{fm}^{3}$, are over-populated. The initial fraction of over-populated cells is found to be bigger at higher energies and in more central collisions. I also find that all QGP cells are over-populated after a couple of

$\mathrm{fm} / c$, and the total transverse area of over-populated cells does not change much during the first few $\mathrm{fm} / c$.

\section{ACKNOWLEDGMENTS}

The author would like to thank B. Betz for valuable discussions and express special thanks to M. Gyulassy for motivating this study. As an external associate, the author thanks the JET Collaboration for help. The author also thanks Dr. X.-N. Wang for updating the JET Collaboration wiki page with the link to the data files from this 
study.

[1] B. Alver and G. Roland, Phys. Rev. C 81, 054905 (2010) [Erratum-ibid. C 82, 039903 (2010)].

[2] J. -P. Blaizot, F. Gelis, J. -F. Liao, L. McLerran and R. Venugopalan, Nucl. Phys. A 873, 68 (2012).

[3] B. Betz and M. Gyulassy, Phys. Rev. C 86, 024903 (2012).

[4] S. Chatrchyan et al. [CMS Collaboration], Phys. Lett. B 718, 795 (2013).

[5] P. Huovinen, P. F. Kolb, U. W. Heinz, P. V. Ruuskanen and S. A. Voloshin, Phys. Lett. B 503, 58 (2001).

[6] B. Betz, J. Noronha, G. Torrieri, M. Gyulassy, I. Mishustin and D. H. Rischke, Phys. Rev. C 79, 034902 (2009).

[7] B. Schenke, S. Jeon and C. Gale, Phys. Rev. Lett. 106, 042301 (2011).

[8] P. Bozek, Phys. Rev. C 85 (2012) 014911.

[9] Z. Xu and C. Greiner, Phys. Rev. C 71, 064901 (2005).

[10] Z. -W. Lin, C. M. Ko, B. -A. Li, B. Zhang and S. Pal, Phys. Rev. C 72, 064901 (2005).

[11] W. Cassing and E. L. Bratkovskaya, Nucl. Phys. A 831, 215 (2009).

[12] H. Petersen, J. Steinheimer, G. Burau, M. Bleicher and H. Stocker, Phys. Rev. C 78, 044901 (2008).

[13] K. Werner, I. .Karpenko, T. Pierog, M. Bleicher and K. Mikhailov, Phys. Rev. C 82, 044904 (2010).

[14] H. Song, S. A. Bass, U. Heinz, T. Hirano and C. Shen, Phys. Rev. Lett. 106, 192301 (2011) [Erratum-ibid. 109, 139904 (2012)].

[15] Z. -W. Lin and C. M. Ko, Phys. Rev. C 65, 034904 (2002).

[16] B. Zhang, C. M. Ko, B. -A. Li and Z. -W. Lin, Phys. Rev. C 61, 067901 (2000).

[17] Z. -W. Lin, S. Pal, C. M. Ko, B. -A. Li and B. Zhang, Phys. Rev. C 64, 011902 (2001).

[18] T. Sjostrand, Comput. Phys. Commun. 82, 74 (1994).

[19] J. Xu and C. M. Ko, Phys. Rev. C 83, 034904 (2011). 
[20] Evolution data files at http://myweb.ecu.edu/linz/ampt/evolutiondata/.

[21] The JET Collaboration wiki page at https://sites.google.com/a/lbl.gov/jetwiki/codepackages/hydro-evolution/.

[22] B. Betz and M. Gyulassy, arXiv:1305.6458 [nucl-th].

[23] K. M. Burke, A. Buzzatti, N. Chang, C. Gale, M. Gyulassy, U. Heinz, S. Jeon and A. Majumder et al., arXiv:1312.5003 [nucl-th].

[24] B. Abelev et al. [ALICE Collaboration], Phys. Rev. C 88, 044910 (2013).

[25] A. Adare et al. [PHENIX Collaboration], Phys. Rev. C 87, 034911 (2013).

[26] B. Abelev et al. [ALICE Collaboration], Phys. Rev. C 88, 044909 (2013).

[27] S. S. Adler et al. [PHENIX Collaboration], Phys. Rev. C 69, 034909 (2004).

[28] I. G. Bearden et al. [BRAHMS Collaboration], Phys. Rev. Lett. 94, 162301 (2005).

[29] Y. Gu [PHENIX Collaboration], Nucl. Phys. A 904-905, 353c (2013).

[30] G. Aad et al. [ATLAS Collaboration], Phys. Rev. C 86, 014907 (2012).

[31] B. Zhang, L. -W. Chen and C. M. Ko, J. Phys. G 35, 065103 (2008).

[32] W. Florkowski and R. Ryblewski, Phys. Rev. C 83, 034907 (2011).

[33] M. Martinez and M. Strickland, Nucl. Phys. A 848, 183 (2010).

[34] D. Bazow, U. W. Heinz and M. Strickland, arXiv:1311.6720 [nucl-th].

[35] Z. Xu and C. Greiner, Phys. Rev. C 76, 024911 (2007).

[36] T. Lappi and L. McLerran, Nucl. Phys. A 772, 200 (2006).

[37] Z. W. Lin, C. M. Ko and S. Pal, Phys. Rev. Lett. 89, 152301 (2002).

[38] J. Berges and D. Sexty, Phys. Rev. Lett. 108, 161601 (2012).

[39] Topical Collaboration on Jet and Electromagnetic Tomography of Extreme Phases of Matter in Heavy-ion Collisions at http://jet.lbl.gov/. 